\title{
Applying Four-Steps Characteristic Ion Filtering with HPLC-QE Orbitrap MS Approach to Rapid Characterize Compound Structure $s$ and Determine Major Representative Components in Modified Tabusen-2 Decoction
}

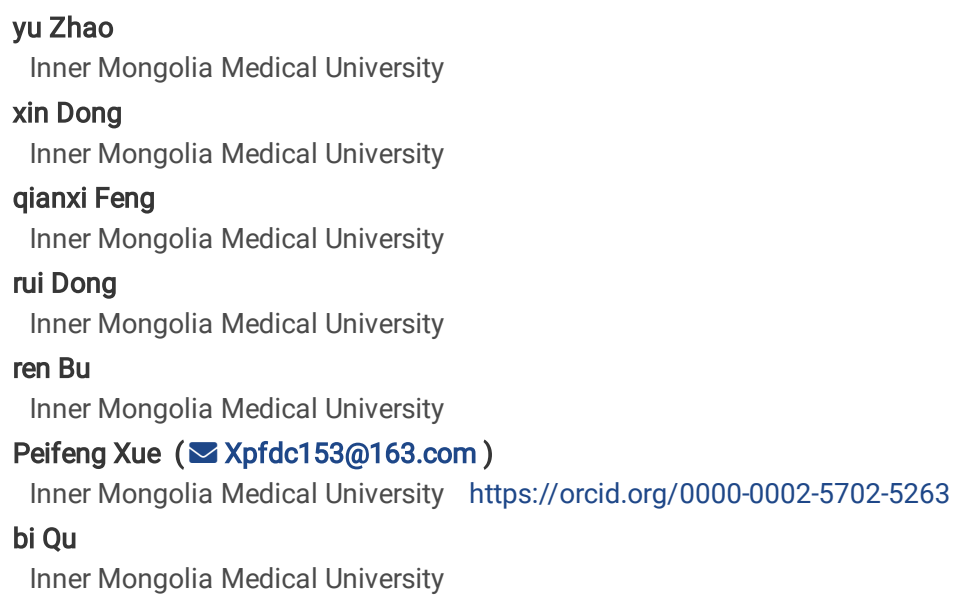




\section{Abstract}

Background: Modified Tabusen-2 decoction (MTBD) is Traditional Chinese Mongolia Medicine, it is consist of "Lancitou" "Duzhong" "Sanqi" and "Honghua". MTBD is mainly used to treat osteoporosis. However, the precise material basis of this prescription is not yet fully elucidated, which affects its modernization development

Methods: We establish a new HPLC-QE Orbitrap MS technology with four-steps characteristic ion filtering (FSCIF) strategy to quickly and effectively identify the structural features of MTBD, and determine the representative compounds content. Then the quantitative method of nineteen main components in MTBD were built, and eight batches MTBD were determined as well.

Results: By using the FSCIF strategy, a total of 143 compounds were unambiguously or tentatively identified, including 5 compounds were first reported in MTBD. Nineteen representative components were simultaneously quantified by HPLC-QE Orbitrap MS, and it is suitable for eight batches of MTBD. Methodology analysis showed that the assay method of 19 compounds (including three pairs of isomers) with a good repeatability, accuracy, and stability.

Conclusion: The method established above was successfully applied to assess the quality of MTBD extracts, which prove that the concentrations of 19 compounds varied significantly in different batches of MTBD extracts. Collectively, our findings enhance our molecular understanding of the MTBD formulation and will allow us to control its quality in a better way. At the same time, this study can promote the development and utilization of ethnic medicine.

\section{Introduction}

Modified Tabusen-2 decoction (MTBD), is developed on the basis of Tubson-2 decoction (TBD), which consist of "Lancitou" (inflorescences of Echinops latifolius Tausch (ELT)), "Duzhong" (barks of Eucommia ulmoides Oliver (EU)), "Sanqi" (roots of Panax notoginseng (PN)) and "Honghua" (flowers of Carthamus tinctorius L. (CT)) [1]. The Mongolian nationality is called a nation on horseback, osteoporosis as a common disease, especially common in the Mongolian nationality. TBD is a traditional classic prescription, it has been used to treat osteoporosis for centuries[2]. On this basis, MTBD has a better therapeutic effect, it mainly has the effects of promoting blood circulation, dispersing healing, reducing swelling and relieving pain, and renewing tendons. The chemical compositions of each herb are various, which has different pharmacological effects acconding to past reports. ELT, a traditional Mongolia herb, contained iochlorogenic acid A (ICGAA), chlorogenic acid and other phenylpropanoids [3, 4], which are the main active components in herb. The pharmacological mitigation of ELT on osteoporosis of postmenopausal women was also reported [5]. EU is enriched with lignans and iridoids, including geniposidic acid (GPA) and pinoresinol diglucoside (PDG), which has obvious antihypertensive effect [6]. In recent years, EU has attracted considerable attention because of its antiosteoporosis, anti-senile dementia, anti-aging, anti-inflammatory, antithrombotic, and antitumor activities [7, 8]. The flavonoids are the main active components of CT, with the efficacy of promoting blood circulation, removing blood stasis and relieving pain [9, 10]. Varieties of natural pigments isolated from CT, such as yellow pigments and red pigments [11], not only have pharmacological functions, but also have some nutritive value. Furthermore, triterpenoid saponins are main active constituents in PN, which are widely used for promoting blood clotting, relieving swelling, and alleviating pain $[12,13]$. Although there have been many reports on the compounds in these medicinal materials, it is not enough to explain the overall structure of MTBD due to the interaction between temperature and herbs in the process of decoction. The chemical components of traditional Chinese medicine are the key to the study of its pharmacological activity, so in order to promote the development of traditional prescriptions, this research is aims to establish a new HPLC-QE Orbitrap MS technology to quickly identify the structural features of MTBD.

In accord with Traditional Chinese medicine (TCM), Traditional Mongolia medicine (TMM) is characterized with multiple components, multiple targets and played different roles in clinical therapy. This means that it is a great challenge to explain the main chemical composition of MTBD by traditional analytical methods. Especially, the irrelevant interference information makes it more difficult to identify the microscale detection. In order to solve this problem, some researchers have used the methods of mass defect, relative mass defect, neutral loss filtering (NLF), mass defect filtering and precursor ion to characterize the chemical structure in TCM or TMM prescription [14-18]. It has vital- important reference value for our following experiment. With the promotion of highresolution mass spectrometry $[19,20]$, we propose a FSCIF strategy for substructure recognition, which can significantly improve the detection effectiveness, accuracy, and sensibility. This analysis program shows obvious efficiency (reduce data processing time) and intelligence (simplify the process of structural identification).

Nineteen representative components were selected for quantitative study. Methodology analysis showed that the assay method of nineteen compounds (including three pairs of isomers) with a good repeatability and stability. In terms of good linearity, acceptable recoveries and reproducibility, the method established above was successfully applied to quality assessment of eight batches MTBD extracts. The result prove that the concentrations of nineteen compounds varied significantly in different batches of MTBD extracts, which could facilitate the better development of quality evaluation technique in MTBD and will help to further its quality control exploration. This experiment explored the material basis of MTBD, promoted the development and utilization of traditional classic prescriptions, and provided new ideas for the analysis of complex prescriptions.

\section{Methods \\ Materials and reagents}

A total four batches of ELT were collected from various areas of Inner Mongolia (including Hohhot, Ordos, Xilingol, Ulan Hot) in August 2020. Different batches of EU, CT and PN powder were purchased from Bozhou Pharmaceutical Co. Ltd. (Anhui, China) and Guoda drugstore (Hohhot, Inner Mongolia). All herbs were authenticated by Professor Bi Qu. These speciments were preserved in the Department of general investigation of traditional Chinese medicine resources, Inner Mongolia Medical University. 
Isochlorogenic acid A (ICGAA), 1,5-dicaffeoylqunic acid (1,5-DQA), genistein (GE), apigenin (APG), luteolin (LT), kaempferol (KPF), quercetin (QC), apigenin-7-0glucuronide (A-7-0-G), rutin (RU), hydroxysafflor yellow $A$ (HSYA), notoginsenoside $R_{1}\left(N G-R_{1}\right)$, ginsenoside $R e(G-R E)$, ginsenoside $R g_{1}\left(G-R G_{1}\right)$, ginsenoside $\mathrm{Rb}_{1}\left(\mathrm{G}-\mathrm{RB}_{1}\right)$, caffeic acid (CA), ferulic acid (FA), geniposidic acid (GPA), chlorogenic acid (CGA), pinoresinol diglucoside (PDG) and digoxin (internal standard, IS) were purchased from Cybertech Limited (Beijing, China), with HPLC purity $\geq 98 \%$. The chemical structures of these 19 compounds were displayed in Fig. 1. LC-MS grade methanol, acetonitrile and formic acid were achieved from Fisher Scientific (Hampton, NH, USA). Deionized water was prepared on a Millipore water purification system (Billerica, MA, USA).

\section{MTBD Sample and standard solutions preparation}

Sample preparation was a critical step for precise and convincing detection by the HPLC-QE Orbitrap mass method. The MTBD samples were prepared under the mature extraction process, and the whole operation process was in line with the basic operation safety regulations of the laboratory. EU, ELT and CT herbal materials were powdered and sieved through a 40-mesh for later extraction. A total $3.6 \mathrm{~g}$ of MTBD powders were accurately weighed (including $1.6 \mathrm{~g}$ of EU, 1.2 $\mathrm{g}$ of ELT, $0.8 \mathrm{~g}$ of CT) and placed in a $250 \mathrm{~mL}$ round-bottomed flask. These powders were immersed in $50 \mathrm{~mL}$ ethanol: water (6:4, V/V) mixture and weighed, then reflux extracted twice, $90 \mathrm{~min}$ for each reflux. Taking into account the recovery rate of PN powder, add $0.4 \mathrm{~g}$ PN before the last extraction. After merging and mixing, the solution was filtered through a $0.45 \mu \mathrm{m}$ microporous membrane. This filtrate was diluted 40 times for HPLC-QE Orbitrap mass injection.

ICGAA 20.05 mg, 1,5-DQA 11.92 mg, GE 4.03 mg, APG 2.15 mg, LT 4.03 mg, KPF 1.30 mg, QC 2.02 mg, A-7-0-G 3.85 mg, RU 4.23 mg, HSYA 19.80 mg, NG-R 10.02 mg, G-Re 19.40 mg, G-Rg 10.17 mg, G-Rb 20.49 mg, CA 2.07 mg, FA 1.05 mg, GPA 4.05 mg, CGA 18.90 mg, PDG 23.40 mg were accurately weighted and transferred into $2 \mathrm{~mL}$ volumetric flask, respectively. Owing to the solubility of these compounds, methanol was applied to prepare the standard solution. In order to improve the precision and accuracy of the content, digoxin was selected as the internal standard. These standard solutions were diluted with mobile phase to final concentration before injection into HPLC-QE Orbitrap mass.

\section{Chromatography and mass spectrometry conditions}

The characterization and quantification of MTBD sample extracts were analyzed using a Thermo HPLC-QE Orbitrap mass system (HPLC, UltiMate 3000, mass system, Quadrupole Exactive Orbitrap $\left.{ }^{\mathrm{T}}\right)$. The qualitative analytical conditions were as follows: HPLC column, COSMOSIL C18 (250mm $\times 4.6 \mathrm{~mm}$ ID, $\left.5 \mu \mathrm{m}\right)$; Solvent system, methanol $(A)$ and water containing $0.1 \%(v / v)$ formic acid (B); Gradient program, 0-5 min, 2\%-5\%A; 5-10 min, 5\%-10\%A; $10-15$ min, 10\%-18\%A; 15-25 min, 18\%-23\%A; 25-35 min, 23\%-28\%A; 35-55 min, 28\%-33\%A; 55-60 min, 33\%-39\%A; 60-70 min, 39\%-43\%A; 70-75 min, 43\%-46\%A; 75$85 \mathrm{~min}, 46 \%-60 \% \mathrm{~A} ; 85-100 \mathrm{~min}, 60 \%-65 \% \mathrm{~A} ; 100-105 \mathrm{~min}, 65 \%-75 \% \mathrm{~A} ; 105-110 \mathrm{~min}, 75 \%-100 \% \mathrm{~A} ; 110-130 \mathrm{~min}, 100 \%-100 \% \mathrm{~A}$. Flow rate, 0.6 mL/ min. Column temperature, $30^{\circ} \mathrm{C}$. Sample injection volume, $10 \mu \mathrm{L}$. The quantitative analysis of MTBD sample extracts was separated on an ACE C18-PFP $(100 \times 3.0 \mathrm{~mm}$ ID, $3 \mu \mathrm{m})$ column. The mobile phase consisted of methanol $(\mathrm{A})$ and water containing $0.3 \%(\mathrm{v} / \mathrm{v})$ formic acid $(B)$. A gradient program was used as follows: 0-6 min, 40\%-40\%A; 6-15 min, 40\%-90\%A; 15-16 min, 90\%-10\%A; 16-21 min, 10\%-10\%A; 21-22 min, 10\%-40\%A; 22-25 min, 40\%-40\%A. The flow rate was set as $0.3 \mathrm{~mL} / \mathrm{min}$. The column temperature was kept at $30^{\circ} \mathrm{C}$. Sample injection volume was $2 \mu \mathrm{L}$.

The qualitative and quantitative mass parameters conditions were set up as follows: Aus gas heater temperature, $150^{\circ} \mathrm{C}$; Capillary temperature, $350^{\circ} \mathrm{C}$; Spray voltage, $3.5 \mathrm{kv}$; S-lens RF level, 50; Sheath gas flow rate, $40 \mathrm{~L}$; Aux gas flow rate, $2 \mathrm{~L}$. Scanning range, $100-1500 \mathrm{~m} / \mathrm{z}$. Mass spectrometry uses full scan mode for analysis in positive ion mode and negative ion mode.

\section{Method Validation}

The dependent variable was the ratio of the peak area of each analyte to the peak area of the internal standard, while the independent variable was set as the concentration value of each analyte, the least square regression was used to construct the standard curve equation. The lower limit of quantification (LLOQ) should be with signal-to-noise ratio $>10$. The precisions and accuracies of intra-day and inter-day was assessed by analyzing each concentration level (low, medium, and high) of six repeated QC samples on the same day and three consecutive days, respectively. Sample stability was investigated after the extracts kept at room temperature for $0 \mathrm{~h}, 6 \mathrm{~h}, 12 \mathrm{~h}, 24 \mathrm{~h}$. Add the mixed control solution equal to the content of each analyte in the sample to the MTBD sample, repeat the preparation of 6 solutions, and calculate the recovery according to the following formula.

Recovery $(\%)=($ detected amount - original amount $) /$ spiked amount $\times 100 \%$

\section{Construction of the identification strategy}

Each type of compounds has its similar core and skeleton. On this basis, the characteristic ion will be produced, which provides us with new ideas for identifying these structures. In addition, FSCIF is especially suitable for compounds with the same structural type contained similar fragmentation pathways with some characteristic ions. Correspondingly, a FSCIF-based and sub-structure scanning strategy will be used for rapid identification of MTBD structures. The analytical strategy is show in Fig. 2. The compounds in MTBD were characterized by HPLC-QE Orbitrap MS technology with FSCIF strategy, which including following steps: 1) established the self-building chemical database of MTBD according to literature and online database; 2) comprehensively summarized characteristic ions for each compound type to conduct global identification of the ingredients in MTBD; 3 ) rapidly screened relevant structure information by neutral loss fragments (NLF) to conform the sugar type, conjunction position, and other information; 4) concluded the precise compound structure through high-precision MS ${ }^{n}$ data. The typical total ion chromatograms (TICs) of MTBD by HPLC-QE Orbitrap mass system in positive and negative ion modes were shown in Fig. 3. 143 compounds were identified through high-precision MS ${ }^{n}$ data, including 51 triterpene saponins, 28 flavanoids, 20 phenylpropanines, 15 iridoids, 12 lignans, 11 polyphenols, and 6 other types (Table 1), in which 5 compounds were first reported in MTBD and 20 compounds were unambiguously identified by comparing with reference standards. These 143 components structures were shown in Figure S1.

Page $3 / 25$ 


\begin{tabular}{|c|c|c|c|c|c|c|c|c|}
\hline No & $\operatorname{tR}(\min )$ & Formula & Identification & $\begin{array}{l}\text { Precursor } \\
\text { ions } \\
(\mathrm{m} / \mathrm{z})\end{array}$ & Diff & $\begin{array}{l}\text { Fragment } \\
(\mathrm{m} / \mathrm{z})\end{array}$ & Type & $\begin{array}{l}\text { Reference } \\
\text { standard }\end{array}$ \\
\hline 1 & 0.25 & $\mathrm{C}_{27} \mathrm{H}_{30} \mathrm{O}_{16}$ & Rutin & $\begin{array}{l}\text { 609.1461[M- } \\
\mathrm{H}]^{-}\end{array}$ & -3.414 & $\begin{array}{l}301.0351, \\
300.0278, \\
283.0325, \\
271.0251, \\
255.0292, \\
227.0321, \\
151.0293\end{array}$ & $\mathrm{~F}$ & Yes \\
\hline 2 & 1.05 & $\mathrm{C}_{15} \mathrm{H}_{10} \mathrm{O}_{7}$ & Quercetin & $\begin{array}{l}301.0353[\mathrm{M}- \\
\mathrm{H}]^{-}\end{array}$ & 3.724 & $\begin{array}{c}273.0405, \\
257.0452, \\
229.0500, \\
178.9978, \\
151.0026 \\
121.0283 \\
107.0126\end{array}$ & $\mathrm{~F}$ & \\
\hline 3 & 1.68 & $\mathrm{C}_{8} \mathrm{H}_{8} \mathrm{O}_{4}$ & Vanillic acid & $\begin{array}{l}167.0349[\mathrm{M}- \\
\mathrm{H}]^{-}\end{array}$ & 0.867 & $\begin{array}{c}152.0105 \\
123.0438 \\
108.0203\end{array}$ & PO & \\
\hline 4 & 2.35 & $\mathrm{C}_{17} \mathrm{H}_{20} \mathrm{O}_{9}$ & Methyl chlorogenic acid & $\begin{array}{l}367.1034[\mathrm{M}- \\
\mathrm{H}]^{-}\end{array}$ & 5.239 & $\begin{array}{c}191.0553, \\
173.0078\end{array}$ & $\mathrm{P}$ & \\
\hline 5 & 3.57 & $\mathrm{C}_{11} \mathrm{H}_{14} \mathrm{O}_{5}$ & Genipin & $\begin{array}{l}225.0768[\mathrm{M}- \\
\mathrm{H}]^{-}\end{array}$ & 2.738 & $\begin{array}{c}207.0659, \\
147.0441, \\
123.0439 \\
101.0231\end{array}$ & I & Yes \\
\hline 6 & 3.82 & $\mathrm{C}_{6} \mathrm{H}_{6} \mathrm{O}_{3}$ & Pyrogallic acid & $\begin{array}{l}125.0244[\mathrm{M}- \\
\mathrm{H}]^{-}\end{array}$ & -1.604 & $\begin{array}{l}\text { 107.4741, } \\
97.0282\end{array}$ & PO & \\
\hline 7 & 4.09 & $\mathrm{C}_{16} \mathrm{H}_{22} \mathrm{O}_{10}$ & Geniposidic acid & $\begin{array}{l}373.1140[\mathrm{M}- \\
\mathrm{H}]^{-}\end{array}$ & -6.227 & $\begin{array}{c}211.0940, \\
193.0498, \\
167.0703, \\
149.0598 \\
123.0439\end{array}$ & I & \\
\hline 8 & 4.47 & $\mathrm{C}_{16} \mathrm{H}_{22} \mathrm{O}_{11}$ & Deacetyl trifolate & $\begin{array}{l}389.1089[\mathrm{M}- \\
\mathrm{H}]^{-}\end{array}$ & 3.167 & $\begin{array}{c}227.0550, \\
209.0356, \\
191.0553, \\
183.0655, \\
165.0541, \\
147.0285, \\
139.0389\end{array}$ & I & \\
\hline 9 & 4.85 & $\mathrm{C}_{10} \mathrm{H}_{14} \mathrm{O}_{10}$ & 2-Methylsuccinyl-6'-0-glucoside & $\begin{array}{l}293.0514[\mathrm{M}- \\
\mathrm{H}]^{-}\end{array}$ & 6.047 & 131.0450 & PO & \\
\hline
\end{tabular}

F: Flavonoids, P: Phenolic acids, T: Triterpenoid saponins, L: Lignans, I: Iridoids, PO: Polyphenol, O: Other compounds

* first reported in MTBD 


\begin{tabular}{|c|c|c|c|c|c|c|c|c|}
\hline No & $\operatorname{tR}(\min )$ & Formula & Identification & $\begin{array}{l}\text { Precursor } \\
\text { ions } \\
(\mathrm{m} / \mathrm{z})\end{array}$ & Diff & $\begin{array}{l}\text { Fragment } \\
(m / z)\end{array}$ & Type & $\begin{array}{l}\text { Reference } \\
\text { standard }\end{array}$ \\
\hline 10 & 4.92 & $\mathrm{C}_{15} \mathrm{H}_{22} \mathrm{O}_{9}$ & Aucubin & $\begin{array}{l}345.1191[\mathrm{M}- \\
\mathrm{H}^{-}\end{array}$ & 2.335 & $\begin{array}{l}\text { 183.0660, } \\
\\
165.0543, \\
139.0391, \\
121.0285\end{array}$ & I & \\
\hline 11 & 5.37 & $\mathrm{C}_{17} \mathrm{H}_{26} \mathrm{O}_{11}$ & Harpagide acetate & $\begin{array}{l}405.1402[\mathrm{M}- \\
\mathrm{H}]^{-}\end{array}$ & -1.364 & $\begin{array}{c}191.0554 \\
147.0289 \\
119.0026 \\
101.0023\end{array}$ & I & \\
\hline 12 & 6.23 & $\mathrm{C}_{15} \mathrm{H}_{10} \mathrm{O}_{6}$ & Kaempferol & $\begin{array}{l}285.0404[\mathrm{M}- \\
\mathrm{H}]^{-}\end{array}$ & 4.615 & $\begin{array}{c}257.0453, \\
243.1601 \\
239.1650 \\
229.0322 \\
199.0395 \\
185.0420\end{array}$ & $\mathrm{~F}$ & Yes \\
\hline 13 & 7.41 & $\mathrm{C}_{23} \mathrm{H}_{34} \mathrm{O}_{15}$ & Genipin gentian diglycoside & $\begin{array}{l}549.1824[\mathrm{M}- \\
\mathrm{H}]^{-}\end{array}$ & 5.378 & $\begin{array}{c}387.2035 \\
207.1128 \\
179.0551 \\
147.0298\end{array}$ & I & \\
\hline 14 & 7.50 & $\mathrm{C}_{25} \mathrm{H}_{24} \mathrm{O}_{11}$ & 3-Caffeoyl-5-coumaroyl-quinic acid & $\begin{array}{l}499.12458[\mathrm{M}- \\
\mathrm{H}]^{-}\end{array}$ & 5.81 & $\begin{array}{l}353.1080 \\
191.0554\end{array}$ & $\mathrm{P}$ & \\
\hline 15 & 8.32 & $\mathrm{C}_{19} \mathrm{H}_{18} \mathrm{O}_{11}$ & Isomangiferin & $\begin{array}{l}421.0776[\mathrm{M}- \\
\mathrm{H}]^{-}\end{array}$ & -1.966 & 259.0224 & $\mathrm{~F}$ & \\
\hline 16 & 8.44 & $\mathrm{C}_{9} \mathrm{H}_{6} \mathrm{O}_{3}$ & Umbelliferone & $\begin{array}{l}161.0244[\mathrm{M}- \\
\mathrm{H}]^{-}\end{array}$ & -0.249 & $\begin{array}{l}135.0441 \\
99.0438 \\
71.0124\end{array}$ & $\mathrm{P}$ & \\
\hline 17 & 8.99 & $\mathrm{C}_{7} \mathrm{H}_{6} \mathrm{O}_{5}$ & Gallic acid & $\begin{array}{l}169.0142[\mathrm{M}- \\
\mathrm{H}]^{-}\end{array}$ & 0.179 & $\begin{array}{l}125.0232 \\
141.0914\end{array}$ & PO & Yes \\
\hline 18 & 9.25 & $\mathrm{C}_{6} \mathrm{H}_{6} \mathrm{O}_{4}$ & 2-hydroxyphenol & $\begin{array}{l}141.0193[\mathrm{M}- \\
\mathrm{H}]^{-}\end{array}$ & 3.247 & 123.0175 & $\mathrm{PO}$ & \\
\hline 19 & 9.76 & $\mathrm{C}_{15} \mathrm{H}_{14} \mathrm{O}_{6}$ & L-Epicatechin & $\begin{array}{l}289.0717[\mathrm{M}- \\
\mathrm{H}]^{-}\end{array}$ & 7.333 & $\begin{array}{l}271.0235 \\
245.0411 \\
205.2713 \\
179.0110\end{array}$ & $\mathrm{~F}$ & \\
\hline 20 & 10.56 & $\mathrm{C}_{4} \mathrm{H}_{4} \mathrm{O}_{4}$ & Maleic acid & $\begin{array}{l}115.0036[\mathrm{M}- \\
\mathrm{H}]^{-}\end{array}$ & -0.479 & 71.0124 & PO & \\
\hline
\end{tabular}

F: Flavonoids, P: Phenolic acids, T: Triterpenoid saponins, L: Lignans, I: Iridoids, PO: Polyphenol, O: Other compounds

* first reported in MTBD

Page 5/25 


\begin{tabular}{|c|c|c|c|c|c|c|c|c|}
\hline No & $\operatorname{tR}(\min )$ & Formula & Identification & $\begin{array}{l}\text { Precursor } \\
\text { ions } \\
(\mathrm{m} / \mathrm{z})\end{array}$ & Diff & $\begin{array}{l}\text { Fragment } \\
(\mathrm{m} / \mathrm{z})\end{array}$ & Type & $\begin{array}{l}\text { Reference } \\
\text { standard }\end{array}$ \\
\hline 21 & 11.43 & $\mathrm{C}_{15} \mathrm{H}_{24} \mathrm{O}_{10}$ & Harpagide & $\begin{array}{l}363.1296[\mathrm{M}- \\
\mathrm{H}]^{-}\end{array}$ & 0.977 & $\begin{array}{l}\text { 183.0652, } \\
89.0228\end{array}$ & I & \\
\hline 22 & 12.53 & $\mathrm{C}_{16} \mathrm{H}_{18} \mathrm{O}_{9}$ & Chlorogenic acid & $\begin{array}{l}353.0878[\mathrm{M}- \\
\mathrm{H}^{-}\end{array}$ & 0.854 & $\begin{array}{l}191.0554, \\
179.0341, \\
173.0446, \\
161.0234, \\
155.0338, \\
137.0322, \\
135.0440 \\
93.0333\end{array}$ & $\mathrm{P}$ & Yes \\
\hline 23 & 14.13 & $\mathrm{C}_{8} \mathrm{H}_{8} \mathrm{O}_{4}$ & Methyl protocatechuic acid & $\begin{array}{l}167.0349[\mathrm{M}- \\
\mathrm{H}^{-}\end{array}$ & 0.508 & $\begin{array}{c}152.0106 \\
123.0439 \\
108.0203\end{array}$ & PO & \\
\hline 24 & 15.84 & $\mathrm{C}_{8} \mathrm{H}_{8} \mathrm{O}_{4}$ & Isovanillic acid & $\begin{array}{l}167.0349[\mathrm{M}- \\
\mathrm{H}^{-}\end{array}$ & -2.536 & 123.0439 & PO & \\
\hline 25 & 18.23 & $\mathrm{C}_{13} \mathrm{H}_{16} \mathrm{O}_{9}$ & Protocatechuic acid-4-glucoside & $\begin{array}{l}315.0721[\mathrm{M}- \\
\mathrm{H}^{-}\end{array}$ & -1.392 & 108.0204 & PO & \\
\hline 26 & 19.56 & $\mathrm{C}_{14} \mathrm{H}_{18} \mathrm{O}_{9}$ & 4-glucopyranoxy-3-benzoic acid & $\begin{array}{l}329.0878[\mathrm{M}- \\
\mathrm{H}^{-}\end{array}$ & 4.340 & $\begin{array}{c}167.0340 \\
152.0105 \\
123.0439 \\
108.0204\end{array}$ & 0 & \\
\hline 27 & 20.23 & $\mathrm{C}_{9} \mathrm{H}_{12} \mathrm{O}_{5}$ & Rehmaglutin C & $\begin{array}{l}199.0611[\mathrm{M}- \\
\mathrm{H}^{-}\end{array}$ & 3.316 & $\begin{array}{l}155.0704 \\
137.0596\end{array}$ & 1 & \\
\hline 28 & 20.39 & $\mathrm{C}_{18} \mathrm{H}_{24} \mathrm{O}_{12}$ & Asperuloside acid & $\begin{array}{l}431.1194[\mathrm{M}- \\
\mathrm{H}^{-}\end{array}$ & -3.114 & $\begin{array}{l}269.0198 \\
251.0098\end{array}$ & I & \\
\hline 29 & 21.77 & $\mathrm{C}_{16} \mathrm{H}_{18} \mathrm{O}_{9}$ & Neochlorogenic acid & $\begin{array}{l}353.0878[\mathrm{M}- \\
\mathrm{H}^{-}\end{array}$ & -0.699 & $\begin{array}{l}191.0554 \\
179.0341 \\
135.0440\end{array}$ & $\mathrm{P}$ & \\
\hline 30 & 22.36 & $\mathrm{C}_{16} \mathrm{H}_{18} \mathrm{O}_{9}$ & 4-caffeoylquinic acid & $\begin{array}{l}353.0878[\mathrm{M}- \\
\mathrm{H}^{-}\end{array}$ & 3.941 & $\begin{array}{l}191.0554 \\
179.0340 \\
135.1440\end{array}$ & $\mathrm{P}$ & \\
\hline 31 & 23.28 & $\mathrm{C}_{20} \mathrm{H}_{24} \mathrm{O}_{7}$ & Cycloolivil & $\begin{array}{l}375.1449[\mathrm{M}- \\
\mathrm{H}^{-}\end{array}$ & -2.075 & $\begin{array}{l}327.1343 \\
297.1207 \\
257.1132 \\
151.0752\end{array}$ & L & \\
\hline 32 & 24.28 & $\mathrm{C}_{9} \mathrm{H}_{8} \mathrm{O}_{4}$ & Caffeic acid & $\begin{array}{l}179.0349[\mathrm{M}- \\
\mathrm{H}^{-}\end{array}$ & 0.856 & 135.0440 & $P$ & Yes \\
\hline 33 & 24.36 & $\mathrm{C}_{9} \mathrm{H}_{10} \mathrm{O}_{4}$ & Dihydro caffeic acid & $\begin{array}{l}181.0506[\mathrm{M}- \\
\mathrm{H}^{-}\end{array}$ & 1.849 & $\begin{array}{c}163.0390 \\
135.0441 \\
119.0488\end{array}$ & $\mathrm{P}$ & \\
\hline
\end{tabular}

F: Flavonoids, P: Phenolic acids, T: Triterpenoid saponins, L: Lignans, I: Iridoids, PO: Polyphenol, O: Other compounds

* first reported in MTBD

Page 6/25 


\begin{tabular}{|c|c|c|c|c|c|c|c|c|}
\hline No & $\operatorname{tR}(\min )$ & Formula & Identification & $\begin{array}{l}\text { Precursor } \\
\text { ions } \\
(m / z)\end{array}$ & Diff & $\begin{array}{l}\text { Fragment } \\
(\mathrm{m} / \mathrm{z})\end{array}$ & Type & $\begin{array}{l}\text { Reference } \\
\text { standard }\end{array}$ \\
\hline 34 & 26.50 & $\mathrm{C}_{7} \mathrm{H}_{6} \mathrm{O}_{4}$ & Gentianic acid & $\begin{array}{l}\text { 153.0193[M- } \\
\mathrm{H}^{-}\end{array}$ & -0.295 & 109.0282 & PO & \\
\hline 35 & 27.23 & $\mathrm{C}_{7} \mathrm{H}_{6} \mathrm{O}_{4}$ & Protocatechuic acid & $\begin{array}{l}153.0193[\mathrm{M}- \\
\mathrm{H}]^{-}\end{array}$ & 0.685 & $\begin{array}{l}109.0283 \\
91.0175\end{array}$ & PO & \\
\hline 36 & 28.03 & $\mathrm{C}_{42} \mathrm{H}_{70} \mathrm{O}_{12}$ & Ginsenoside $\mathrm{F}_{4}$ & $\begin{array}{l}789.4759[\mathrm{M} \\
+\mathrm{Na}^{+}\end{array}$ & -1.837 & $\begin{array}{l}707.1499 \\
643.4222 \\
349.1090\end{array}$ & T & \\
\hline 37 & 28.55 & $\mathrm{C}_{20} \mathrm{H}_{24} \mathrm{O}_{7}$ & Oleoresin & $\begin{array}{l}375.1449[\mathrm{M}- \\
\mathrm{H}^{-}\end{array}$ & -1.018 & $\begin{array}{c}179.0341 \\
161.0233\end{array}$ & L & \\
\hline 38 & 29.40 & $\mathrm{C}_{16} \mathrm{H}_{18} \mathrm{O}_{9}$ & Cryptochlorogenic acid & $\begin{array}{l}353.0878[\mathrm{M}- \\
\mathrm{H}]^{-}\end{array}$ & 3.516 & $\begin{array}{c}191.0554 \\
179.0340 \\
173.0446 \\
135.0440\end{array}$ & $\mathrm{P}$ & \\
\hline 39 & 30.90 & $\mathrm{C}_{20} \mathrm{H}_{24} \mathrm{O}_{7}$ & Olivil & $\begin{array}{l}375.1449[\mathrm{M}- \\
\mathrm{H}]^{-}\end{array}$ & -2.635 & $\begin{array}{c}327.1360 \\
195.1251 \\
179.0341 \\
161.0220\end{array}$ & L & \\
\hline 40 & 32.26 & $\mathrm{C}_{17} \mathrm{H}_{24} \mathrm{O}_{10}$ & Geniposide & $\begin{array}{l}387.1296[\mathrm{M}- \\
\mathrm{H}]^{-}\end{array}$ & 3.128 & $\begin{array}{c}207.1025 \\
123.0444 \\
101.0232\end{array}$ & 1 & \\
\hline 41 & 33.49 & $\mathrm{C}_{33} \mathrm{H}_{44} \mathrm{O}_{19}$ & Naringin dihydrochalcone 4-O- $\beta$ - D-glucoside & $\begin{array}{l}743.2404[\mathrm{M}- \\
\mathrm{H}^{-}\end{array}$ & 3.128 & $\begin{array}{c}373.1295 \\
313.1088 \\
181.0498 \\
151.0396\end{array}$ & $\mathrm{~F}$ & \\
\hline 42 & 34.26 & $\mathrm{C}_{27} \mathrm{H}_{32} \mathrm{O}_{16}$ & Hydroxysafflor yellow A & $\begin{array}{l}\text { 611.1617[M- } \\
\mathrm{H}^{-}\end{array}$ & 2.207 & $\begin{array}{l}491.1200 \\
473.1092 \\
403.1042 \\
325.0720\end{array}$ & $\mathrm{~F}$ & Yes \\
\hline 43 & 37.58 & $\mathrm{C}_{10} \mathrm{H}_{10} \mathrm{O}_{4}$ & Ferulic acid & $\begin{array}{l}193.0506[\mathrm{M}- \\
\mathrm{H}^{-}\end{array}$ & 2.208 & $\begin{array}{c}178.0264 \\
149.0598 \\
134.0362\end{array}$ & $\mathrm{P}$ & Yes \\
\hline 44 & 38.11 & $\mathrm{C}_{32} \mathrm{H}_{42} \mathrm{O}_{16}$ & Pinoresinol diglucoside & $\begin{array}{l}681.2400[\mathrm{M}- \\
\mathrm{H}^{-}\end{array}$ & 2.039 & $\begin{array}{c}519.5070 \\
357.1346 \\
151.0390 \\
136.0159\end{array}$ & L & \\
\hline 45 & 39.59 & $\mathrm{C}_{16} \mathrm{H}_{18} \mathrm{O}_{8}$ & 3-0-p-coumaroylquinic acid & $\begin{array}{l}337.0928[\mathrm{M}- \\
\mathrm{H}^{-}\end{array}$ & 6.688 & $\begin{array}{c}191.0553 \\
173.0448 \\
163.0390\end{array}$ & $P$ & \\
\hline 46 & 40.21 & $\mathrm{C}_{27} \mathrm{H}_{36} \mathrm{O}_{13}$ & Citrusin B & $\begin{array}{l}567.2083[\mathrm{M}- \\
\mathrm{H}]^{-}\end{array}$ & -4.289 & $\begin{array}{l}341.1384 \\
329.1394\end{array}$ & L & \\
\hline
\end{tabular}

F: Flavonoids, P: Phenolic acids, T: Triterpenoid saponins, L: Lignans, I: Iridoids, PO: Polyphenol, O: Other compounds

* first reported in MTBD

Page $7 / 25$ 


\begin{tabular}{|c|c|c|c|c|c|c|c|c|}
\hline No & $\operatorname{tR}(\min )$ & Formula & Identification & $\begin{array}{l}\text { Precursor } \\
\text { ions } \\
(m / z)\end{array}$ & Diff & $\begin{array}{l}\text { Fragment } \\
(\mathrm{m} / \mathrm{z})\end{array}$ & Type & $\begin{array}{l}\text { Reference } \\
\text { standard }\end{array}$ \\
\hline 47 & 41.77 & $\mathrm{C}_{27} \mathrm{H}_{30} \mathrm{O}_{17}$ & $\begin{array}{l}\text { Quercetin-3, 4'-0-di- } \beta \text {-glu } \\
\text { copyranoside }\end{array}$ & $\begin{array}{l}625.1410[\mathrm{M}- \\
\mathrm{H}]^{-}\end{array}$ & 0.831 & $\begin{array}{l}463.0884 \\
301.0350 \\
271.0243\end{array}$ & $\mathrm{~F}$ & \\
\hline 48 & 42.23 & $\mathrm{C}_{15} \mathrm{H}_{26} \mathrm{O}_{9}$ & Eucommioside & $\begin{array}{l}349.1504[\mathrm{M}- \\
\mathrm{H}^{-}\end{array}$ & -1.102 & $\begin{array}{l}187.1528, \\
89.0230\end{array}$ & I & \\
\hline 49 & 43.69 & $\mathrm{C}_{10} \mathrm{H}_{10} \mathrm{O}_{3}$ & Coniferyl aldehyde & $\begin{array}{l}177.0557[\mathrm{M}- \\
\mathrm{H}]^{-}\end{array}$ & -0.101 & 162.0312 & $P$ & \\
\hline 50 & 46.23 & $\mathrm{C}_{20} \mathrm{H}_{22} \mathrm{O}_{7}$ & Erythroglycerin- $\beta$ - terpineol aldehyde ether & $\begin{array}{l}373.1292[\mathrm{M}- \\
\mathrm{H}^{-}\end{array}$ & 4.259 & $\begin{array}{c}177.0548 \\
165.0547 \\
150.0308\end{array}$ & $\mathrm{P}$ & \\
\hline 51 & 48.38 & $\mathrm{C}_{26} \mathrm{H}_{28} \mathrm{O}_{16}$ & Quercetin 3-0-Sambu bioside & $\begin{array}{l}595.1305[\mathrm{M} \\
+\mathrm{H}]^{+}\end{array}$ & -0.638 & 301.0327 & $\mathrm{~F}$ & \\
\hline 52 & 49.62 & $\mathrm{C}_{26} \mathrm{H}_{32} \mathrm{O}_{11}$ & Pinoresinol-4'-O- $\beta$-D-glucopyranoside & $\begin{array}{l}519.1871[\mathrm{M}- \\
\mathrm{H}^{-}\end{array}$ & -3.929 & $\begin{array}{l}357.1345, \\
151.0390\end{array}$ & L & \\
\hline 53 & 54.63 & $\mathrm{C}_{26} \mathrm{H}_{32} \mathrm{O}_{11}$ & Pinoresinol- $\beta$-D-glucoside; & $\begin{array}{l}519.1871[\mathrm{M}- \\
\mathrm{H}^{-}\end{array}$ & 3.334 & $\begin{array}{l}357.1345, \\
342.1107, \\
311.1293, \\
151.0390 \\
136.0154\end{array}$ & L & \\
\hline 54 & 55.16 & $\mathrm{C}_{21} \mathrm{H}_{20} \mathrm{O}_{12}$ & Isoquercitrin & $\begin{array}{l}463.0881[\mathrm{M}- \\
\mathrm{H}]^{-}\end{array}$ & 3.623 & $\begin{array}{l}301.0349 \\
271.0321 \\
255.0299\end{array}$ & $\mathrm{~F}$ & Yes \\
\hline 55 & 56.44 & $\mathrm{C}_{22} \mathrm{H}_{28} \mathrm{O}_{14}$ & 5-(3'- o-caffeoylglucosyl) quinine & $\begin{array}{l}515.1406[\mathrm{M}- \\
\mathrm{H}]^{-}\end{array}$ & 8.420 & $\begin{array}{l}191.0555, \\
161.0234 \\
135.0440\end{array}$ & $P$ & \\
\hline 56 & 57.45 & $\mathrm{C}_{22} \mathrm{H}_{28} \mathrm{O}_{14}$ & 1-0- (3 '- o-caffeoylglucosyl) quinine & $\begin{array}{l}515.1406[\mathrm{M}- \\
\mathrm{H}^{-}\end{array}$ & 2.499 & $\begin{array}{l}179.0341 \\
173.0446 \\
161.0233 \\
135.0440\end{array}$ & $P$ & \\
\hline $57 *$ & 57.73 & $\mathrm{C}_{33} \mathrm{H}_{40} \mathrm{O}_{21}$ & $\begin{array}{l}\text { Quercetin 3-glucosyl- (1->3) -rhamnosyl- (1->6) - } \\
\text { galactoside }\end{array}$ & $\begin{array}{l}771.1989[\mathrm{M}- \\
\mathrm{H}^{-}\end{array}$ & 2.070 & $\begin{array}{l}609.1469 \\
463.0873 \\
301.0351\end{array}$ & $\mathrm{~F}$ & \\
\hline 58 & 58.66 & $\mathrm{C}_{28} \mathrm{H}_{36} \mathrm{O}_{13}$ & Syringaresionl-O- $\beta$-D-g1uc0pyranoside & $\begin{array}{l}579.2083[\mathrm{M}- \\
\mathrm{H}^{-}\end{array}$ & 3.298 & 417.1557 & $P$ & \\
\hline 59 & 59.50 & $\mathrm{C}_{35} \mathrm{H}_{60} \mathrm{O}_{6}$ & Daucosterol & $\begin{array}{l}575.4317[\mathrm{M}- \\
\mathrm{H}^{-}\end{array}$ & 2.329 & 397.7564 & $\mathrm{~T}$ & \\
\hline 60 & 62.59 & $\mathrm{C}_{25} \mathrm{H}_{24} \mathrm{O}_{12}$ & 1,5-dicaffeoylqunic acid & $\begin{array}{l}515.1194[\mathrm{M}- \\
\mathrm{H}^{-}\end{array}$ & 2.499 & $\begin{array}{c}353.0881, \\
191.0554, \\
135.0440\end{array}$ & $P$ & \\
\hline
\end{tabular}

F: Flavonoids, P: Phenolic acids, T: Triterpenoid saponins, L: Lignans, I: Iridoids, PO: Polyphenol, O: Other compounds

* first reported in MTBD 


\begin{tabular}{|c|c|c|c|c|c|c|c|c|}
\hline No & $\operatorname{tR}(\min )$ & Formula & Identification & $\begin{array}{l}\text { Precursor } \\
\text { ions } \\
(m / z)\end{array}$ & Diff & $\begin{array}{l}\text { Fragment } \\
(\mathrm{m} / \mathrm{z})\end{array}$ & Type & $\begin{array}{l}\text { Reference } \\
\text { standard }\end{array}$ \\
\hline 61 & 63.34 & $\mathrm{C}_{25} \mathrm{H}_{24} \mathrm{O}_{12}$ & Isochlorogenic acid A & $\begin{array}{l}515.1194[\mathrm{M}- \\
\mathrm{H}^{-}\end{array}$ & 1.288 & $\begin{array}{l}353.0881 \\
191.0554 \\
179.0341 \\
173.0446 \\
135.0440\end{array}$ & $\mathrm{P}$ & \\
\hline 62 & 64.00 & $\mathrm{C}_{42} \mathrm{H}_{72} \mathrm{O}_{15}$ & $\begin{array}{l}\text { 6- O- } \beta \text {-D-glucopyranosyl-20-o- } \beta \text {-D-glucopyranosyl- } \\
3 \beta, 6 \beta, 12 \beta, 20 \text { (S)7-25-pentaphydroxydammar-23- } \\
\text { eneDroginsenoside } \mathrm{Rg}_{1}\end{array}$ & $\begin{array}{l}839.4763[\mathrm{M} \\
+\mathrm{Na}]^{+}\end{array}$ & -2.644 & 659.4114 & T & \\
\hline 63 & 65.10 & $\mathrm{C}_{25} \mathrm{H}_{24} \mathrm{O}_{12}$ & Isochlorogenic acid B & $\begin{array}{l}515.1194[\mathrm{M}- \\
\mathrm{H}^{-}\end{array}$ & 1.288 & $\begin{array}{l}353.0881 \\
335.0777 \\
191.0554 \\
179.0341 \\
173.0446\end{array}$ & $P$ & \\
\hline 64 & 66.70 & $\mathrm{C}_{9} \mathrm{H}_{16} \mathrm{O}_{4}$ & Eucommitol & $\begin{array}{l}187.0975[\mathrm{M}- \\
\mathrm{H}]^{-}\end{array}$ & 1.521 & $\begin{array}{l}169.0861 \\
143.1068 \\
125.0960\end{array}$ & 1 & Yes \\
\hline 65 & 66.72 & $\mathrm{C}_{6} \mathrm{H}_{4} \mathrm{O}_{4}$ & Coumalic acid & $\begin{array}{l}139.0036[\mathrm{M}- \\
\mathrm{H}^{-}\end{array}$ & 6.332 & 119.5097 & 0 & \\
\hline 66 & 67.26 & $\mathrm{C}_{18} \mathrm{H}_{16} \mathrm{O}_{5}$ & Sideroxylin & $\begin{array}{l}311.0924[\mathrm{M}- \\
\mathrm{H}^{-}\end{array}$ & 2.177 & 267.0663 & $\mathrm{~F}$ & \\
\hline 67 & 67.68 & $\mathrm{C}_{21} \mathrm{H}_{20} \mathrm{O}_{12}$ & Hyperoside & $\begin{array}{l}463.0882[\mathrm{M}- \\
\mathrm{H}]^{-}\end{array}$ & 4.206 & $\begin{array}{l}301.03455 \\
151.00258\end{array}$ & $\mathrm{~F}$ & \\
\hline 68 & 68.78 & $\mathrm{C}_{15} \mathrm{H}_{26} \mathrm{O}_{7}$ & $\begin{array}{l}\text { 2-(5-hydroxyethyl-2,3-dimethyl-2-cyclopenten-1-yl)- } \\
\text { glucopyranoside }\end{array}$ & $\begin{array}{l}317.1605[\mathrm{M}- \\
\mathrm{H}^{-}\end{array}$ & 4.580 & $\begin{array}{l}243.1238 \\
225.1132\end{array}$ & I & \\
\hline 69 & 69.40 & $\mathrm{C}_{21} \mathrm{H}_{20} \mathrm{O}_{10}$ & Apigenin-7-0-glucuronide & $\begin{array}{l}431.0983[\mathrm{M}- \\
\mathrm{H}^{-}\end{array}$ & 3.147 & 269.0376 & $\mathrm{~F}$ & \\
\hline 70 & 70.29 & $\mathrm{C}_{9} \mathrm{H}_{16} \mathrm{O}_{3}$ & 1-deoxyeucommitol & $\begin{array}{l}171.1026[\mathrm{M}- \\
\mathrm{H}]^{-}\end{array}$ & 0.930 & $\begin{array}{l}127.1118 \\
125.0959\end{array}$ & 1 & \\
\hline 71 & 72.63 & $\mathrm{C}_{21} \mathrm{H}_{20} \mathrm{O}_{11}$ & Astragalin & $\begin{array}{l}447.0933[\mathrm{M}- \\
\mathrm{H}^{-}\end{array}$ & 2.532 & $\begin{array}{l}285.0395 \\
241.0829 \\
217.0886\end{array}$ & $\mathrm{~F}$ & \\
\hline 72 & 73.55 & $\mathrm{C}_{21} \mathrm{H}_{18} \mathrm{O}_{11}$ & Baicalin & $\begin{array}{l}445.0776[\mathrm{M}- \\
\mathrm{H}]^{-}\end{array}$ & 4.724 & 269.0456 & $\mathrm{~F}$ & \\
\hline 73 & 74.23 & $\mathrm{C}_{27} \mathrm{H}_{30} \mathrm{O}_{15}$ & Nicotiflorin & $\begin{array}{l}593.1511[\mathrm{M}- \\
\mathrm{H}^{-}\end{array}$ & 3.681 & $\begin{array}{l}285.0404 \\
255.0307 \\
227.0352\end{array}$ & $\mathrm{~F}$ & \\
\hline 74 & 74.88 & $\mathrm{C}_{27} \mathrm{H}_{30} \mathrm{H}_{15}$ & Safflor yellow (A) & $\begin{array}{l}593.1511[\mathrm{M}- \\
\mathrm{H}^{-}\end{array}$ & 3.884 & 285.0404 & $\mathrm{~F}$ & Yes \\
\hline 75 & 75.61 & $\mathrm{C}_{18} \mathrm{H}_{14} \mathrm{O}_{6}$ & Milletenin C & $\begin{array}{l}325.0717[\mathrm{M}- \\
\mathrm{H}]^{-}\end{array}$ & 2.650 & 310.0848 & $\mathrm{~F}$ & \\
\hline 76 & 76.26 & $\mathrm{C}_{12} \mathrm{H}_{16} \mathrm{O}_{3}$ & 3-Butyl-4-hydroxy-4,5-dihydro-2-benzofuran-1(3H)-one & $\begin{array}{l}207.1026[\mathrm{M}- \\
\mathrm{H}^{-}\end{array}$ & 0.368 & 135.0443 & 0 & \\
\hline
\end{tabular}

F: Flavonoids, P: Phenolic acids, T: Triterpenoid saponins, L: Lignans, I: Iridoids, PO: Polyphenol, O: Other compounds

* first reported in MTBD 


\begin{tabular}{|c|c|c|c|c|c|c|c|c|}
\hline No & $\operatorname{tR}(\min )$ & Formula & Identification & $\begin{array}{l}\text { Precursor } \\
\text { ions } \\
(m / z)\end{array}$ & Diff & $\begin{array}{l}\text { Fragment } \\
(\mathrm{m} / \mathrm{z})\end{array}$ & Type & $\begin{array}{l}\text { Reference } \\
\text { standard }\end{array}$ \\
\hline 77 & 77.03 & $\mathrm{C}_{11} \mathrm{H}_{12} \mathrm{O}_{4}$ & Ethyl caffeate & $\begin{array}{l}207.0662[\mathrm{M}- \\
\mathrm{H}]^{-}\end{array}$ & 3.162 & $\begin{array}{c}179.0341 \\
161.0234 \\
135.0440\end{array}$ & $\mathrm{P}$ & \\
\hline 78 & 77.96 & $\mathrm{C}_{48} \mathrm{H}_{82} \mathrm{O}_{19}$ & Notoginsenoside $\mathrm{R}_{6}$ & $\begin{array}{l}985.5342[\mathrm{M} \\
+\mathrm{Na}^{+}\end{array}$ & -2.049 & $\begin{array}{l}365.1045 \\
305.0816\end{array}$ & T & \\
\hline 79 & 78.56 & $\mathrm{C}_{48} \mathrm{H}_{82} \mathrm{O}_{19}$ & Notoginsenoside $\mathrm{R}_{3}$ & $\begin{array}{l}985.5342[\mathrm{M} \\
+\mathrm{Na}]^{+}\end{array}$ & 2.402 & $\begin{array}{l}645.4159, \\
365.1044\end{array}$ & T & \\
\hline $80^{*}$ & 79.20 & $\mathrm{C}_{28} \mathrm{H}_{32} \mathrm{O}_{16}$ & 6-Methoxykaempferol 3-robinobioside & $\begin{array}{l}\text { 623.16175[M- } \\
\mathrm{H}^{-}\end{array}$ & 1.524 & $\begin{array}{l}315.0509 \\
301.0320 \\
300.0276\end{array}$ & $\mathrm{~F}$ & \\
\hline 81* & 79.40 & $\mathrm{C}_{29} \mathrm{H}_{36} \mathrm{O}_{15}$ & 3,4',6'-Trihydroxy-4,2'-dimethoxychalcone 4'-0-rutinoside & $\begin{array}{l}\text { 623.19814[M- } \\
\mathrm{H}^{-}\end{array}$ & 6.18 & $\begin{array}{l}315.0510 \\
301.0313 \\
300.0376\end{array}$ & $\mathrm{~F}$ & \\
\hline 82 & 79.58 & $\mathrm{C}_{48} \mathrm{H}_{82} \mathrm{O}_{19}$ & Notoginsenoside M & $\begin{array}{l}985.5342[\mathrm{M} \\
+\mathrm{Na}]^{+}\end{array}$ & -3.227 & $\begin{array}{l}805.4688 \\
365.1047\end{array}$ & $\mathrm{~T}$ & \\
\hline 83 & 81.76 & $\mathrm{C}_{48} \mathrm{H}_{82} \mathrm{O}_{19}$ & Notoginsenoside $\mathrm{N}$ & $\begin{array}{l}985.5342[\mathrm{M} \\
+\mathrm{Na}^{+}\end{array}$ & -2.983 & 805.4689 & $\mathrm{~T}$ & \\
\hline 84 & 82.77 & $\mathrm{C}_{48} \mathrm{H}_{82} \mathrm{O}_{19}$ & 20-0-glucoginsenoside Rf & $\begin{array}{l}985.5342[\mathrm{M} \\
+\mathrm{Na}^{+}\end{array}$ & -1.684 & $\begin{array}{l}805.4689 \\
365.2320\end{array}$ & T & \\
\hline $85^{*}$ & 83.83 & $\mathrm{C}_{23} \mathrm{H}_{22} \mathrm{O}_{11}$ & Apigenin 7- (2"-acetylglucoside) & $\begin{array}{l}473.1089[\mathrm{M}- \\
\mathrm{H}^{-}\end{array}$ & 1.569 & $\begin{array}{l}413.0891 \\
269.0379\end{array}$ & $\mathrm{~F}$ & \\
\hline 86 & 84.34 & $\mathrm{C}_{41} \mathrm{H}_{68} \mathrm{O}_{12}$ & Notoginsenoside $T_{5}$ & $\begin{array}{l}775.4602[\mathrm{M} \\
+\mathrm{Na}^{+}\end{array}$ & -2.385 & $\begin{array}{l}692.0035, \\
643.3312, \\
463.3556, \\
335.0930\end{array}$ & $\mathrm{~T}$ & \\
\hline 87 & 84.47 & $\mathrm{C}_{47} \mathrm{H}_{80} \mathrm{O}_{18}$ & Notoginsenoside $\mathrm{R}_{1}$ & $\begin{array}{l}931.5271[\mathrm{M}- \\
\mathrm{H}^{-}\end{array}$ & 0.633 & $\begin{array}{l}799.4888 \\
637.4328 \\
475.3800 \\
391.0658\end{array}$ & $\mathrm{~T}$ & Yes \\
\hline 88 & 85.37 & $\mathrm{C}_{15} \mathrm{H}_{10} \mathrm{O}_{6}$ & Luteolin & $\begin{array}{l}285.0404[\mathrm{M}- \\
\mathrm{H}^{-}\end{array}$ & 4.720 & $\begin{array}{l}257.0453 \\
151.0030\end{array}$ & $\mathrm{~F}$ & \\
\hline 89 & 85.76 & $\mathrm{C}_{42} \mathrm{H}_{72} \mathrm{O}_{14}$ & Majoroside $\mathrm{F}_{4}$ & $\begin{array}{l}823.4814[\mathrm{M} \\
+\mathrm{Na}]^{+}\end{array}$ & -1.603 & 643.4166 & $\mathrm{~T}$ & \\
\hline 90 & 86.17 & $\mathrm{C}_{42} \mathrm{H}_{72} \mathrm{O}_{14}$ & 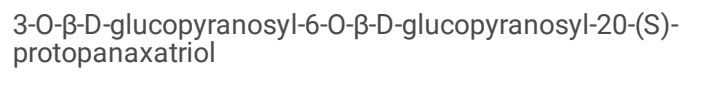 & $\begin{array}{l}823.4814[\mathrm{M} \\
+\mathrm{Na}^{+}\end{array}$ & -3.157 & $\begin{array}{l}703.0069 \\
643.4163\end{array}$ & $\mathrm{~T}$ & \\
\hline 91 & 87.18 & $\mathrm{C}_{42} \mathrm{H}_{72} \mathrm{O}_{14}$ & Gynoside B & $\begin{array}{l}823.4814[\mathrm{M} \\
+\mathrm{Na}]^{+}\end{array}$ & -1.603 & 643.4164 & $\mathrm{~T}$ & \\
\hline 92 & 87.69 & $\mathrm{C}_{42} \mathrm{H}_{72} \mathrm{O}_{14}$ & Ginsenoside $\mathrm{Rg}_{1}$ & $\begin{array}{l}823.4814[\mathrm{M} \\
+\mathrm{Na}^{+}\end{array}$ & -1.603 & 643.4104 & $\mathrm{~T}$ & \\
\hline
\end{tabular}

F: Flavonoids, P: Phenolic acids, T: Triterpenoid saponins, L: Lignans, I: Iridoids, PO: Polyphenol, O: Other compounds

* first reported in MTBD

Page 10/25 


\begin{tabular}{|c|c|c|c|c|c|c|c|c|}
\hline No & $\operatorname{tR}(\min )$ & Formula & Identification & $\begin{array}{l}\text { Precursor } \\
\text { ions } \\
(\mathrm{m} / \mathrm{z})\end{array}$ & Diff & $\begin{array}{l}\text { Fragment } \\
(\mathrm{m} / \mathrm{z})\end{array}$ & Type & $\begin{array}{l}\text { Reference } \\
\text { standard }\end{array}$ \\
\hline 93 & 88.21 & $\mathrm{C}_{45} \mathrm{H}_{74} \mathrm{O}_{17}$ & Malonyl ginsenoside $\mathrm{Rg}_{1}$ & $\begin{array}{l}909.4818[\mathrm{M} \\
+\mathrm{Na}^{+}\end{array}$ & -1.891 & $\begin{array}{l}865.4895 \\
729.4166 \\
685.4270\end{array}$ & $\mathrm{~T}$ & \\
\hline 94 & 89.59 & $\mathrm{C}_{48} \mathrm{H}_{80} \mathrm{O}_{19}$ & Notoginsenoside G & $\begin{array}{l}983.5186[\mathrm{M} \\
+\mathrm{Na}^{+}\end{array}$ & -2.897 & 803.4535 & $\mathrm{~T}$ & \\
\hline $95^{\star}$ & 89.94 & $\mathrm{C}_{30} \mathrm{H}_{28} \mathrm{O}_{12}$ & $\begin{array}{l}\text { 4,2',3',4'-Tetrahydroxychalcone 4'-0- (2"-0-p-coumaroyl) } \\
\text { glucoside }\end{array}$ & $\begin{array}{l}579.1507[\mathrm{M}- \\
\mathrm{H}]^{-}\end{array}$ & 0.622 & $\begin{array}{c}271.0614 \\
151.0027 \\
107.0126\end{array}$ & $\mathrm{~F}$ & \\
\hline 96 & 90.05 & $\mathrm{C}_{15} \mathrm{H}_{10} \mathrm{O}_{5}$ & Genistein & $\begin{array}{l}269.0455[\mathrm{M}- \\
\mathrm{H}^{-}\end{array}$ & 4.572 & $\begin{array}{l}225.0554, \\
201.0555, \\
151.0027 \\
117.0329 \\
107.0124\end{array}$ & $\mathrm{~F}$ & Yes \\
\hline 97 & 90.57 & $\mathrm{C}_{15} \mathrm{H}_{10} \mathrm{O}_{5}$ & Apigenin & $\begin{array}{l}269.0455[\mathrm{M}- \\
\mathrm{H}]^{-}\end{array}$ & 4.572 & $\begin{array}{l}225.0555, \\
201.0553 \\
151.0025 \\
117.0328 \\
107.0124\end{array}$ & $\mathrm{~F}$ & Yes \\
\hline 98 & 91.24 & $\mathrm{C}_{41} \mathrm{H}_{70} \mathrm{O}_{13}$ & Pseudoginsenoside $\mathrm{RT}_{3}$ & $\begin{array}{l}793.4708[\mathrm{M} \\
+\mathrm{Na}^{+}\end{array}$ & 3.882 & 613.4072 & $\mathrm{~T}$ & \\
\hline 99 & 91.79 & $\mathrm{C}_{44} \mathrm{H}_{74} \mathrm{O}_{15}$ & Yesanchinoside D & $\begin{array}{l}865.4919[\mathrm{M} \\
+\mathrm{Na}^{+}\end{array}$ & -4.633 & 685.4267 & $\mathrm{~T}$ & \\
\hline 100 & 92.47 & $\mathrm{C}_{30} \mathrm{H}_{26} \mathrm{O}_{12}$ & Apigenin-7-0-(6"-coumaroyl) glucosid & $\begin{array}{l}577.1351[\mathrm{M}- \\
\mathrm{H}]^{-}\end{array}$ & 1.390 & $\begin{array}{l}431.0988 \\
269.0457\end{array}$ & $\mathrm{~F}$ & \\
\hline 101 & 92.96 & $\mathrm{C}_{20} \mathrm{H}_{22} \mathrm{O}_{6}$ & Epipinoresinol & $\begin{array}{l}357.1343[\mathrm{M}- \\
\mathrm{H}]^{-}\end{array}$ & 1.216 & $\begin{array}{c}151.1533 \\
136.0809 \\
121.0282\end{array}$ & $\mathrm{~L}$ & \\
\hline 102 & 94.52 & $\mathrm{C}_{42} \mathrm{H}_{72} \mathrm{O}_{14}$ & Ginsenoside Rf & $\begin{array}{l}823.4814[\mathrm{M} \\
+\mathrm{Na}]^{+}\end{array}$ & -2.113 & $\begin{array}{l}661.5368 \\
641.4468 \\
365.1043\end{array}$ & $\mathrm{~T}$ & \\
\hline 103 & 95.39 & $\mathrm{C}_{41} \mathrm{H}_{70} \mathrm{O}_{13}$ & Notoginsenoside $\mathrm{R}_{2}$ & $\begin{array}{l}793.4708[\mathrm{M} \\
+\mathrm{Na}^{+}\end{array}$ & -2.583 & $\begin{array}{c}661.4249 \\
481.3630 \\
335.0939\end{array}$ & $\mathrm{~T}$ & \\
\hline 104 & 95.89 & $\mathrm{C}_{42} \mathrm{H}_{72} \mathrm{O}_{13}$ & Ginsenoside $\mathrm{Rg}_{2}$ & $\begin{array}{l}807.4865[\mathrm{M} \\
+\mathrm{Na}]^{+}\end{array}$ & -1.362 & $\begin{array}{l}661.4281, \\
481.3676, \\
349.1101\end{array}$ & $\mathrm{~T}$ & \\
\hline 105 & 96.47 & $\mathrm{C}_{36} \mathrm{H}_{62} \mathrm{O}_{9}$ & Gypenoside LXXVI & $\begin{array}{l}661.4286[\mathrm{M} \\
+\mathrm{Na}^{+}\end{array}$ & 2.479 & $\begin{array}{l}601.2890 \\
481.3620\end{array}$ & $\mathrm{~T}$ & \\
\hline 106 & 97.28 & $\mathrm{C}_{36} \mathrm{H}_{62} \mathrm{O}_{9}$ & Ginsenoside $\mathrm{Rh}_{1}$ & $\begin{array}{l}661.4286[\mathrm{M} \\
\left.+\mathrm{Na}^{+}\right]^{+}\end{array}$ & -1.769 & $\begin{array}{l}481.3650 \\
413.2539\end{array}$ & $\mathrm{~T}$ & \\
\hline
\end{tabular}

F: Flavonoids, P: Phenolic acids, T: Triterpenoid saponins, L: Lignans, I: Iridoids, PO: Polyphenol, O: Other compounds

* first reported in MTBD

Page $11 / 25$ 


\begin{tabular}{|c|c|c|c|c|c|c|c|c|}
\hline No & $\operatorname{tR}(\min )$ & Formula & Identification & $\begin{array}{l}\text { Precursor } \\
\text { ions } \\
(\mathrm{m} / \mathrm{z})\end{array}$ & Diff & $\begin{array}{l}\text { Fragment } \\
(\mathrm{m} / \mathrm{z})\end{array}$ & Type & $\begin{array}{l}\text { Reference } \\
\text { standard }\end{array}$ \\
\hline 107 & 98.42 & $\mathrm{C}_{59} \mathrm{H}_{100} \mathrm{O}_{27}$ & Ginsenoside $\mathrm{Ra}_{3}$ & $\begin{array}{l}1263.6344[\mathrm{M} \\
+\mathrm{Na}]^{+}\end{array}$ & -2.327 & $\begin{array}{c}\text { 789.4784, } \\
497.1457 \\
437.1239\end{array}$ & $\mathrm{~T}$ & \\
\hline 108 & 99.42 & $\mathrm{C}_{59} \mathrm{H}_{100} \mathrm{O}_{27}$ & Notoginsenoside Fa & $\begin{array}{l}1263.6344[\mathrm{M} \\
+\mathrm{Na}]^{+}\end{array}$ & -0.688 & 921.5158 & $\mathrm{~T}$ & \\
\hline 109 & 100.51 & $\mathrm{C}_{54} \mathrm{H}_{92} \mathrm{O}_{22}$ & Notoginsenoside I & $\begin{array}{l}1115.5972[\mathrm{M} \\
+\mathrm{Na}]^{+}\end{array}$ & -1.470 & $\begin{array}{l}\text { 773.4795, } \\
365.1046\end{array}$ & $\mathrm{~T}$ & \\
\hline 110 & 101.91 & $\mathrm{C}_{54} \mathrm{H}_{92} \mathrm{O}_{23}$ & Ginsenoside $\mathrm{Rb}_{1}$ & $\begin{array}{l}1107.5956[\mathrm{M}- \\
\mathrm{H}]^{-}\end{array}$ & 0.589 & $\begin{array}{l}945.5432 \\
783.4906 \\
621.4368 \\
459.3851\end{array}$ & $\mathrm{~T}$ & Yes \\
\hline 111 & 102.94 & $\mathrm{C}_{42} \mathrm{H}_{72} \mathrm{O}_{13}$ & Ginsenoside $\mathrm{Rg}_{3}$ & $\begin{array}{l}807.4865[\mathrm{M} \\
+\mathrm{Na}]^{+}\end{array}$ & -0.904 & 365.1046 & $\mathrm{~T}$ & \\
\hline 112 & 103.85 & $\mathrm{C}_{48} \mathrm{H}_{82} \mathrm{O}_{18}$ & Ginsenoside Re & $\begin{array}{l}969.5393[\mathrm{M} \\
+\mathrm{Na}]^{+}\end{array}$ & -1.908 & 789.4742 & $\mathrm{~T}$ & Yes \\
\hline 113 & 105.07 & $\mathrm{C}_{54} \mathrm{H}_{92} \mathrm{O}_{23}$ & Yesanchinoside $\mathrm{E}$ & $\begin{array}{l}1131.5921[\mathrm{M} \\
+\mathrm{Na}]^{+}\end{array}$ & -5.768 & $\begin{array}{c}789.4737 \\
365.1045\end{array}$ & $\mathrm{~T}$ & \\
\hline 114 & 106.08 & $\mathrm{C}_{38} \mathrm{H}_{64} \mathrm{O}_{10}$ & 6'-O-acetyl ginsenoside $F_{1}$ & $\begin{array}{l}703.4391[\mathrm{M} \\
+\mathrm{Na}]^{+}\end{array}$ & 0.071 & 481.3647 & $\mathrm{~T}$ & \\
\hline 115 & 106.58 & $\mathrm{C}_{56} \mathrm{H}_{94} \mathrm{O}_{24}$ & Quinquenoside $\mathrm{R}_{1}$ & $\begin{array}{l}1173.6027[\mathrm{M} \\
+\mathrm{Na}]^{+}\end{array}$ & -2.548 & $\begin{array}{l}831.4845 \\
365.1044\end{array}$ & $\mathrm{~T}$ & \\
\hline 116 & 107.65 & $\mathrm{C}_{56} \mathrm{H}_{94} \mathrm{O}_{24}$ & 6"'-O-acetylginsenoside $\mathrm{Rb}_{1}$ & $\begin{array}{l}1173.6027[\mathrm{M} \\
+\mathrm{Na}]^{+}\end{array}$ & -2.326 & $\begin{array}{l}831.4845 \\
789.4744 \\
407.1151 \\
347.0945\end{array}$ & $\mathrm{~T}$ & \\
\hline 117 & 108.75 & $\mathrm{C}_{53} \mathrm{H}_{90} \mathrm{O}_{22}$ & Ginsenoside $\mathrm{Rb}_{2}$ & $\begin{array}{l}1101.5815[\mathrm{M} \\
+\mathrm{Na}]^{+}\end{array}$ & -1.479 & $\begin{array}{l}\text { 789.4740, } \\
335.0939\end{array}$ & $\mathrm{~T}$ & \\
\hline 118 & 109.76 & $\mathrm{C}_{53} \mathrm{H}_{90} \mathrm{O}_{22}$ & Notoginsenoside L & $\begin{array}{l}1101.5815[\mathrm{M} \\
+\mathrm{Na}]^{+}\end{array}$ & -1.479 & 789.4740 & $\mathrm{~T}$ & \\
\hline 119 & 110.33 & $\mathrm{C}_{57} \mathrm{H}_{94} \mathrm{O}_{26}$ & Malonyl ginsenoside $\mathrm{Rb}_{1}$ & $\begin{array}{l}1217.5925[\mathrm{M} \\
+\mathrm{Na}]^{+}\end{array}$ & -2.727 & $\begin{array}{l}1173.5993, \\
875.4738, \\
831.4844, \\
789.4738\end{array}$ & $\mathrm{~T}$ & \\
\hline 120 & 111.31 & $\mathrm{C}_{48} \mathrm{H}_{82} \mathrm{O}_{17}$ & Vina-ginsenoside $R_{3}$ & $\begin{array}{l}953.5444[\mathrm{M} \\
+\mathrm{Na}]^{+}\end{array}$ & -2.349 & 773.4788 & $\mathrm{~T}$ & \\
\hline 121 & 111.47 & $\mathrm{C}_{48} \mathrm{H}_{82} \mathrm{O}_{18}$ & Gypenoside XVII & $\begin{array}{l}969.5393[\mathrm{M} \\
+\mathrm{Na}]^{+}\end{array}$ & -0.908 & 365.1048 & $\mathrm{~T}$ & \\
\hline
\end{tabular}

F: Flavonoids, P: Phenolic acids, T: Triterpenoid saponins, L: Lignans, I: Iridoids, PO: Polyphenol, O: Other compounds

* first reported in MTBD

Page $12 / 25$ 


\begin{tabular}{|c|c|c|c|c|c|c|c|c|}
\hline No & $\mathrm{tR}(\min )$ & Formula & Identification & $\begin{array}{l}\text { Precursor } \\
\text { ions } \\
(\mathrm{m} / \mathrm{z})\end{array}$ & Diff & $\begin{array}{l}\text { Fragment } \\
(\mathrm{m} / \mathrm{z})\end{array}$ & Type & $\begin{array}{l}\text { Reference } \\
\text { standard }\end{array}$ \\
\hline 122 & 112.04 & $\mathrm{C}_{57} \mathrm{H}_{94} \mathrm{O}_{26}$ & 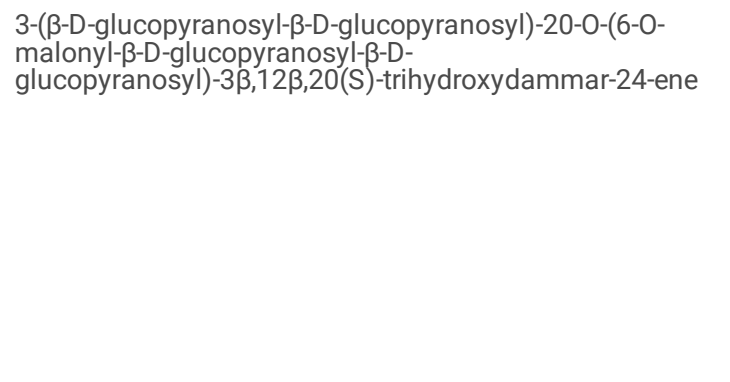 & $\begin{array}{l}1217.5925[\mathrm{M} \\
+\mathrm{Na}]^{+}\end{array}$ & -1.07 & $\begin{array}{l}1173.6008, \\
1131.5912, \\
875.4739 \\
831.4839 \\
789.4733 \\
451.1044 \\
407.1150\end{array}$ & $\mathrm{~T}$ & \\
\hline 123 & 112.53 & $\mathrm{C}_{48} \mathrm{H}_{82} \mathrm{O}_{18}$ & Ginsenoside Rd & $\begin{array}{l}945.5428[\mathrm{M}- \\
\mathrm{H}]^{-}\end{array}$ & 0.857 & $\begin{array}{l}783.4907 \\
621.4375 \\
459.3848 \\
375.3146\end{array}$ & $\mathrm{~T}$ & Yes \\
\hline 124 & 112.57 & $\mathrm{C}_{36} \mathrm{H}_{62} \mathrm{O}_{8}$ & Notoginsenoside $\mathrm{R}_{7}$ & $\begin{array}{l}645.4336[\mathrm{M} \\
+\mathrm{Na}]^{+}\end{array}$ & -3.249 & $\begin{array}{l}627.3813 \\
465.3691\end{array}$ & $\mathrm{~T}$ & \\
\hline 125 & 113.48 & $\begin{array}{l}\mathrm{C}_{36} \mathrm{H}_{60} \mathrm{O}_{8} \\
\mathrm{C} 36 \mathrm{H} 6007\end{array}$ & Ginsenoside $\mathrm{Rh}_{3}$ & $\begin{array}{l}643.4180[\mathrm{M} \\
+\mathrm{Na}]^{+}\end{array}$ & -3.232 & $\begin{array}{l}583.3644 \\
463.3514\end{array}$ & $\mathrm{~T}$ & \\
\hline 126 & 113.89 & $\mathrm{C}_{51} \mathrm{H}_{84} \mathrm{O}_{21}$ & Malonyl ginsenoside Rd & $\begin{array}{l}1055.5397[\mathrm{M} \\
+\mathrm{Na}]^{+}\end{array}$ & 3.598 & $\begin{array}{l}875.4738 \\
789.4740\end{array}$ & $\mathrm{~T}$ & \\
\hline 127 & 114.07 & $\mathrm{C}_{48} \mathrm{H}_{82} \mathrm{O}_{18}$ & Gypenoside LXXII & $\begin{array}{l}969.5393[\mathrm{M} \\
+\mathrm{Na}]^{+}\end{array}$ & -1.691 & 789.4739 & $\mathrm{~T}$ & \\
\hline 128 & 114.88 & $\mathrm{C}_{36} \mathrm{H}_{62} \mathrm{O}_{11}$ & Notoginsenoside $T_{4}$ & $\begin{array}{l}693.4184[\mathrm{M} \\
+\mathrm{Na}]^{+}\end{array}$ & -3.763 & 633.3707 & $\mathrm{~T}$ & \\
\hline 129 & 115.36 & $\mathrm{C}_{47} \mathrm{H}_{80} \mathrm{O}_{17}$ & $\begin{array}{l}\text { 3-O-[ } \beta \text {-D-glucopyranosyl(1-2)- } \beta \text {-D-glucopyranosyl]-20-0- } \beta \text { - } \\
\text { D-xylopyranosyl-3 } \beta, 12 \beta, 20(s) \text {-trihydroxydammar-24-ene }\end{array}$ & $\begin{array}{l}939.5287[\mathrm{M} \\
+\mathrm{Na}]^{+}\end{array}$ & -0.872 & 789.4735 & $\mathrm{~T}$ & \\
\hline 130 & 116.22 & $\mathrm{C}_{15} \mathrm{H}_{10} \mathrm{O}_{5}$ & Baicalein & $\begin{array}{l}269.0455[\mathrm{M}- \\
\mathrm{H}]^{-}\end{array}$ & 4.238 & 197.1905 & $\mathrm{~F}$ & Yes \\
\hline 131 & 117.02 & $\mathrm{C}_{47} \mathrm{H}_{8} \mathrm{OO}_{18}$ & $\begin{array}{l}\text { 6-O-[xylopyranosyl- } \beta \text {-D- } \\
\text { glucopyranosyl]-3 } 3,6 \beta, 12 \beta, 20(s), 25 \text {-pentahydroxydammar }\end{array}$ & $\begin{array}{l}811.4814[\mathrm{M} \\
+\mathrm{Na}]^{+}\end{array}$ & 1.208 & $\begin{array}{c}793.3365 \\
751.2600 \\
679.2239 \\
499.1350 \\
412.1227 \\
335.0018\end{array}$ & $\mathrm{~T}$ & \\
\hline 132 & 117.70 & $\mathrm{C}_{20} \mathrm{H}_{22} \mathrm{O}_{6}$ & Pinoresinol & $\begin{array}{l}357.1343[\mathrm{M}- \\
\mathrm{H}]^{-}\end{array}$ & 1.340 & $\begin{array}{c}313.1811 \\
151.1520 \\
136.0819\end{array}$ & $\mathrm{~L}$ & \\
\hline 133 & 118.87 & $\mathrm{C}_{42} \mathrm{H}_{72} \mathrm{O}_{13}$ & Ginsenoside $F_{2}$ & $\begin{array}{l}807.4865[\mathrm{M} \\
+\mathrm{Na}]^{+}\end{array}$ & 0.458 & 627.4217 & $\mathrm{~T}$ & \\
\hline 134 & 120.08 & $\mathrm{C}_{42} \mathrm{H}_{72} \mathrm{O}_{13}$ & Gypenoside LXXV & $\begin{array}{l}807.4865[\mathrm{M} \\
+\mathrm{Na}]^{+}\end{array}$ & 7.789 & 365.1045 & $\mathrm{~T}$ & \\
\hline 135 & 121.53 & $\mathrm{C}_{29} \mathrm{H}_{42} \mathrm{O}_{5}$ & Ulmoidol & $\begin{array}{l}469.2959[\mathrm{M}- \\
\mathrm{H}]^{-}\end{array}$ & 3.715 & 423.2238 & $\mathrm{~T}$ & \\
\hline 136 & 121.65 & $\mathrm{C}_{28} \mathrm{H}_{34} \mathrm{O}_{4}$ & Unknown & $\begin{array}{l}433.2384[\mathrm{M}- \\
\mathrm{H}]^{-}\end{array}$ & -0.685 & 433.2577 & 0 & \\
\hline
\end{tabular}

F: Flavonoids, P: Phenolic acids, T: Triterpenoid saponins, L: Lignans, I: Iridoids, PO: Polyphenol, O: Other compounds

* first reported in MTBD

Page 13/25 


\begin{tabular}{|c|c|c|c|c|c|c|c|c|}
\hline No & $\operatorname{tR}(\min )$ & Formula & Identification & $\begin{array}{l}\text { Precursor } \\
\text { ions } \\
(m / z)\end{array}$ & Diff & $\begin{array}{l}\text { Fragment } \\
(\mathrm{m} / \mathrm{z})\end{array}$ & Type & $\begin{array}{l}\text { Reference } \\
\text { standard }\end{array}$ \\
\hline 137 & 122.04 & $\mathrm{C}_{36} \mathrm{H}_{60} \mathrm{O}_{9}$ & Ginsenoside $\mathrm{Rh}_{7}$ & $\begin{array}{l}659.4129[\mathrm{M} \\
+\mathrm{Na}^{+}\end{array}$ & -0.349 & 599.3925 & $\mathrm{~T}$ & \\
\hline 138 & 122.50 & $\mathrm{C}_{32} \mathrm{H}_{42} \mathrm{O}_{17}$ & 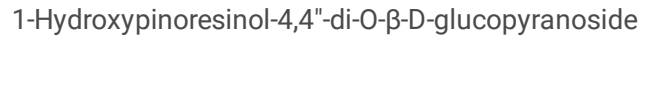 & $\begin{array}{l}697.2349[\mathrm{M}- \\
\mathrm{H}]^{-}\end{array}$ & 0.112 & $\begin{array}{l}535.1532, \\
373.0323\end{array}$ & $\mathrm{~L}$ & \\
\hline 139 & 122.99 & $\mathrm{C}_{20} \mathrm{H}_{24} \mathrm{O}_{8}$ & Threo-dihydroxydehy-drodiconiferyl alcohol & $\begin{array}{l}391.1398[\mathrm{M}- \\
\mathrm{H}^{-}\end{array}$ & -3.313 & $\begin{array}{l}313.1747, \\
295.0882\end{array}$ & L & \\
\hline 140 & 123.91 & $\mathrm{C}_{16} \mathrm{H}_{32} \mathrm{O}_{2}$ & Palmitic acid & $\begin{array}{l}255.2329[\mathrm{M}- \\
\mathrm{H}^{-}\end{array}$ & 1.978 & 241.3251 & 0 & Yes \\
\hline 141 & 124.01 & $\mathrm{C}_{20} \mathrm{H}_{24} \mathrm{O}_{8}$ & Erytho-dihydroxydehydrodiconiferyl alcohol & $\begin{array}{l}391.1398[\mathrm{M}- \\
\mathrm{H}^{-}\end{array}$ & -2.359 & $\begin{array}{l}341.1587 \\
313.0930 \\
207.0832\end{array}$ & L & \\
\hline 142 & 125.83 & $\mathrm{C}_{18} \mathrm{H}_{36} \mathrm{O}_{2}$ & Palmitic acid ethyl ester & $\begin{array}{l}283.2642[\mathrm{M}- \\
\mathrm{H}]^{-}\end{array}$ & 3.859 & 89.0229 & 0 & \\
\hline 143 & 127.10 & $\mathrm{C}_{9} \mathrm{H}_{12} \mathrm{O}_{4}$ & Eucommidiol & $\begin{array}{l}\text { 183.0662[M- } \\
\mathrm{H}^{-}\end{array}$ & 0.268 & $\begin{array}{l}\text { 139.1124, } \\
93.7235\end{array}$ & I & \\
\hline $\mathrm{F}: \mathrm{Flc}$ & vonoids, $\mathrm{P}$ : & Phenolic aci & T: Triterpenoid saponins, L: Lignans, I: Iridoids, PO: PC & ol, O: Other con & ounds & & & \\
\hline * firs & eported ir & MTBD & & & & & & \\
\hline
\end{tabular}

\section{Results}

\section{Identification of triterpenoid saponins}

Triterpenoid saponins were typical bioactive components of PN, which were classified into two categories of protopanaxadiol (PPD) triterpene saponins and protopanaxatriol (PPT) triterpene saponins, the characteristic ions at $m / z 459.39$ [aglycones- $\mathrm{H}^{-}$and at $\mathrm{m} / \mathrm{z} 475.38$ [aglycones- $\left.\mathrm{H}\right]^{-}$, corresponded to the PPD and PPT type ginsenosides [21]. In this study, most triterpene saponins (46 compounds) were detected [M + Na] ${ }^{+}$in positiveion mode, other triterpenoid saponins (5 compounds) were detected $[\mathrm{M}-\mathrm{H}]^{-}$in negative ion mode, excimer ion peaks can produce different cleavage modes to provide structural information such as aglycone type, sugar type and its junction positionand. Compounds $\mathbf{1 1 0}$ and $\mathbf{1 2 3}$ were filtered by characteristic ion $\mathrm{m} / \mathrm{z} \mathbf{4 5 9 . 3 9}$, which tentatively identified PPD type ginsenosides, compound $110\left(\mathrm{C}_{54} \mathrm{H}_{92} \mathrm{O}_{23}\right)[\mathrm{M}-\mathrm{H}]^{-}$at $\mathrm{m} / z 1107.5956$, its molecular ion peak successively lost the four molecules of glucose, and obtained $\mathrm{m} / \mathrm{z}$ 945.5432, $\mathrm{m} / \mathrm{z}$ 783.4906, $\mathrm{m} / \mathrm{z} 621.4368$, and $\mathrm{m} / \mathrm{z}$ 459.3851. Compared with the standard, compound 110 was identified as ginsenoside $\mathrm{Rb}_{1}$, the possible cleavage pathways of ginsenoside $\mathrm{Rb}_{1}$ were shown in Figure S2-A. Similarly, [M-H] ${ }^{-}$at $m / z 945.5428$ (Compound 123 ) was tentatively identified Ginsenoside Rd, the main fragment ions were [M-H-glc] $]^{-} \mathrm{m} / \mathrm{z}$ 783.4907, [M-H-glc-glc] ${ }^{-} \mathrm{m} / \mathrm{z}$ 621.4375, and [M-H-glc-glc-glc] $]^{-} \mathrm{m} / \mathrm{z} 459.3848$ [22]. Compound 87 were filtered by characteristic ion $m / z$ 475.38, which tentatively identified PPT type ginsenoside. In the secondary mass spectrum, fragment ions $m / z$ 799.4888, $\mathrm{m} / z$ 637.4328, $\mathrm{m} / \mathrm{z} 475.3800$ and $\mathrm{m} / \mathrm{z} 391.0658$ were $[\mathrm{M}-\mathrm{H}-\mathrm{xyl}]^{-}$, [M-H-xyl-glc] ${ }^{-}$, [M-H-xyl-glc-glc] ${ }^{-}$and aglycon, the possible cleavage pathways of $[\mathrm{M}-\mathrm{H}]^{-}$were shown in Figure S2-B.

By using the FSCIF strategy, a total of eleven compounds $(107,112,113,116,117,118,119,122,126,127,129)$ were detetcted by characteristic ion of 789.47 $\mathrm{Da}\left(\left[\mathrm{M}+\mathrm{Na} \text {-glcglc }{ }^{6} \text { malony }\right]^{+}\right)$. The retention time of compound 122 was $112.04 \mathrm{~min}$, the fragment ions $\mathrm{m} / \mathrm{z} 451.1044$ and $\mathrm{m} / \mathrm{z} 789.4733 \mathrm{were}$ a pair of complementary ions [glcglc ${ }^{6}$ malonyl $\left.+\mathrm{Na}\right]^{+}$and $\left[\mathrm{M}+\mathrm{Na}\right.$-gicglc ${ }^{6}$ malonyl] ${ }^{+}$. In addition, the fragment ions were observed in $\mathrm{m} / \mathrm{z} 1173.6008,113 \mathrm{l} .5912$, $875.4739,83 \mathrm{l} .4839$ and 407.1150, which were assigned to $\left[\mathrm{M}+\mathrm{Na}-\mathrm{CO}_{2}\right]^{+},[\mathrm{M}+\mathrm{Na}-\text { malonyl }]^{+},[\mathrm{M}+\mathrm{Na}-\mathrm{glcglc}]^{+},\left[\mathrm{M}+\mathrm{Na}-\left(\mathrm{glcglc}+\mathrm{CO}_{2}\right)^{+}\right]$and $\left[\mathrm{glcglc}{ }^{6} \mathrm{malonyl}+\right.$ $\left.\mathrm{Na}-\mathrm{CO}_{2}\right]^{+}$fragment ions. The possible cleavage pathways of compound 122 are shown in Figure S2-C.

Addtionally, the sugar type and its junction positionand were concluded with the application of NLF strategy. The position of sugar fragments on the aglycon was relatively fixed (C3,C6 and C12), and the main types of sugars were glc (162.02 Da), rha (146.01 Da) and xyl (132.02 Da), the linkage between sugars is

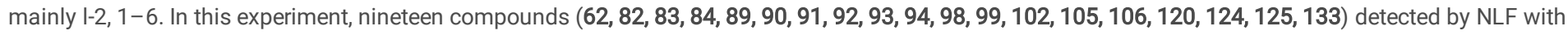
162.02 Da. Compounds 36, 86, 103 and 104 filtered by $146.01 \mathrm{Da}$ or $132.02 \mathrm{Da}$ were obtained. Compound $103\left(\mathrm{C}_{42} \mathrm{H}_{72} \mathrm{O}_{13}\right)[\mathrm{M}+\mathrm{Na}]^{+}$at $m / z 793.4708$ tentatively identified Notoginsenoside $\mathrm{R}_{2}$, the main fragment ions were $[\mathrm{M}-\mathrm{H}-\mathrm{xyl}]^{-} \mathrm{m} / \mathrm{z} 661.4249$, [M-H-xyl-glc $]^{-} \mathrm{m} / z$ 481.3630, and $[\mathrm{M}-\mathrm{H}-\mathrm{xyl}-\mathrm{glc}-\mathrm{rha}]^{-} \mathrm{m} / \mathrm{z}$ 335.0939. Compound $104\left(\mathrm{C}_{42} \mathrm{H}_{72} \mathrm{O}_{13}\right)[\mathrm{M}+\mathrm{Na}]^{+}$at $\mathrm{m} / z 807.4865$ tentatively identified Ginsenoside $\mathrm{Rg}_{2}$. First, the ion at $\mathrm{m} / \mathrm{z} 661.4281 \mathrm{was}$ formed by the neutral loss of a rhamnose unit of the ion at $m / z$ 807.4865. Second, the ion at $m / z 481.3676$ was formed by the neutral loss of a glucose unit of the ion at $\mathrm{m} / \mathrm{z} 661.4281$. Finally, ion at $\mathrm{m} / \mathrm{z} 349.1101$ was formed by the neutral loss of a xylose unit of the ion at $m / z 481.3676$.

Identification of flavonoids 
Most flavonoid aglycones were derivatives of quercetin, kaempferol and apigenin, so we set 301.03 Da, 285.04 Da, 269.04 Da as characteristic ions templates

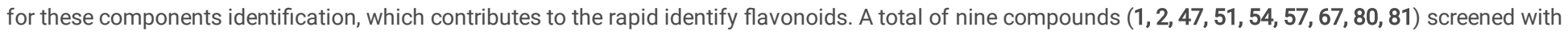
301.03 Da were found, compound 1 showed the $[\mathrm{M}-\mathrm{H}]^{-}$at $\mathrm{m} / \mathrm{z} 609.1461$, which tentatively identified as rutin, its important fragment ion was $301.03 \mathrm{Da}$ in secondary mass spectra, indicating the neutral loss of $308.11 \mathrm{Da}\left(\mathrm{C}_{12} \mathrm{H}_{20} \mathrm{O}_{9}\right)$. In addition, the occurrence of $\mathrm{m} / z 283.0325, \mathrm{~m} / z 255.0292, \mathrm{~m} / z 227.0321 \mathrm{were}$ a better proof [ $\left.\mathrm{M}-\mathrm{H}-\mathrm{C}_{12} \mathrm{H}_{20} \mathrm{O}_{9}-\mathrm{H}_{2} \mathrm{O}\right]^{-}$, [M- $\left.\mathrm{H}-\mathrm{C}_{12} \mathrm{H}_{20} \mathrm{O}_{9}-\mathrm{H}_{2} \mathrm{O}-\mathrm{CO}\right]^{-}$, and [M- $\left.\mathrm{H}_{-} \mathrm{C}_{12} \mathrm{H}_{20} \mathrm{O}_{9}-\mathrm{H}_{2} \mathrm{O}-2 \mathrm{CO}\right]^{-}$, which were the main peak, appeared in second mass spectra

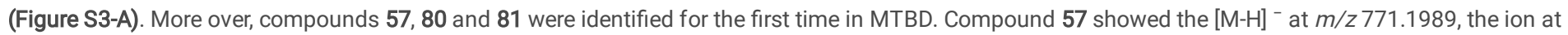
$\mathrm{m} / z 609.1469$ was formed by the neutral loss of a glucose unit of the ion at $\mathrm{m} / z 771.1989$. Besides, the ion at $\mathrm{m} / z 463.0873$ was formed by the neutral loss of axylose unit of the ion at $m / z 609.1469$. Finally, ion at $m / z 301.0351$ was formed by the neutral loss of a glucose unit of the ion at $m / z 463.0873$. Hence, compound 57 was tentatively identified Quercetin 3-glucosyl- (1->3) -rhamnosyl- (1->6) -galactoside.

A total of five compounds $(\mathbf{1 2}, \mathbf{7 1}, \mathbf{7 3}, \mathbf{7 4 , 8 8})$ acquired with 285.04 Da were found. Compound 12 showed the $[\mathrm{M}-\mathrm{H}]^{-}{ }^{-}$at $m / z 285.0404, m / z 257.0453, m / z$ 239.1650, $\mathrm{m} / \mathrm{z} 229.0322$, and $\mathrm{m} / \mathrm{z} 185.0420$, corresponding to [M-H-CO$]^{-}$, [M-H-CO- $\left.\mathrm{H}_{2} \mathrm{O}\right]^{-}$, [M-H-2 $\left.\mathrm{CO}\right]^{-}$, and [M-H-2 $\left.\mathrm{CO}^{-} \mathrm{CO}_{2}\right)^{-}$, which were contribute to the crack of C2-C3 and C4-C10. In addition, the fracture of C4-C10 bond can also lead to the removal of $\mathrm{C}_{2} \mathrm{H}_{2} \mathrm{O}(42.02 \mathrm{Da})$, which was corresponds to $\mathrm{m} / \mathrm{z} 243.1601$. Next, the removal of $\mathrm{CO}_{2}(44.01 \mathrm{Da})$ and result in the generation of $\mathrm{m} / z \mathbf{z} 199.0395$. By using the FSCIF strategy, seven compounds $(\mathbf{6 9}, \mathbf{7 2 , 8 5 , 9 6 , 9 7 , 1 0 0 , 1 3 0 )}$ screened with 269.04 Da were found. Compound 97 was tentatively identified apigenin, a high abundance secondary mass spectrometer fragment ion $m / z$ 225.0555 was formed after $\mathrm{CO}_{2}(44.01 \mathrm{Da})$ loss, indicating that apigenin derivatives were easier to lose $\mathrm{CO}_{2}$. In addition, $\mathrm{m} / z 269.0455$ lost one molecule $\mathrm{C}_{3} \mathrm{O}_{2}$ (68.02 Da), resulting in $\mathrm{m} / z$ 201.0553. Apigenin, which a flavonoid with double bond on the six membered ring, can also undergo ring opening reaction of $C$ ring, resulting in fragment ions such as $m / z 151.0025, m / z 117.0328, m / z 107.0124$. These structural changes were also reflected at a Retro Diels-Alder reaction (RDA) [23]. Hence, the characteristic ion of RDA was set by $151.00 \mathrm{Da}$, compounds $(\mathbf{1}, \mathbf{2}, \mathbf{4 1}, \mathbf{6 7}, \mathbf{8 8}, \mathbf{9 5}, \mathbf{9 6 , 9 7 )}$ screened with $151.00 \mathrm{Da}$ were found. Compound 95 was identified as 4,2',3',4'-Tetrahydroxychalcone 4'-0- (2"-0-p-coumaroyl) glucoside, which was being reported from MTBD for the first time. Its molecular ion peak $m / z 269.0455$ at $[\mathrm{M}-\mathrm{H}]^{-}$was observed, the fragment ions $m / z 271.0614, m / z 151.0027, m / z 107.0126$ were proved that $\left[\mathrm{M}^{-} \mathrm{H}-\mathrm{C}_{15} \mathrm{H}_{16} \mathrm{O}_{7}\right]^{-}$, $\left[\mathrm{M}-\mathrm{H}-\mathrm{C}_{15} \mathrm{H}_{16} \mathrm{O}_{7}-\mathrm{C}_{8} \mathrm{H}_{8} \mathrm{O}\right]^{-}$, and $\left[\mathrm{M}-\mathrm{H}-\mathrm{C}_{15} \mathrm{H}_{16} \mathrm{O}_{7}-\mathrm{C}_{8} \mathrm{H}_{8} \mathrm{O}-\mathrm{C}_{9} \mathrm{H}_{9} \mathrm{O}_{2}\right]^{-}$.

\section{Identification of phenylpropanines}

Phenylpropanoids and their derivatives, including monocaffeoylquinic acids, biscaffeoylquinic acids and acaffeoylquinic acid derivatives, were main components widely present in MTBD. Some papers [24] have previously shown that phenylpropanoids have multifaceted effects which include antiinflammatory, antioxidant, antimicrobial, antidiabetic activities as well as exhibit renoprotective, hepatoprotective, and cardioprotective effects. By using the FSCIF strategy, twenty phenylpropanines were found, the ions at $m / z 191.05 \mathrm{Da}$ and $179.03 \mathrm{Da}$ represented the base peaks of quinic acid, whereas ions at $\mathrm{m} / \mathrm{z}$ 161.02 Da and 135.04 Da represented the base peaks of caffeic acid. Chlorogenic acid is an ester of caffeic acid and quinic acid, which indicates that

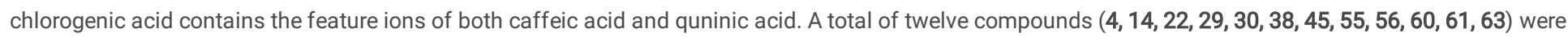
detected by $m / z 191.05 \mathrm{Da}$ and $179.03 \mathrm{Da}$. Compound 22 was tentatively identified chlorogenic acids, produce $\mathrm{m} / z$ 191.0554, $\mathrm{m} / z 179.0341$ (Compound 32 ), $m / z 173.0446, m / z 161.0234, m / z 155.0338, m / z 137.0322, m / z 135.0440$ and $m / z$ 93.0333, which were corresponding to $\left[\mathrm{M}^{-} \mathrm{H}-\mathrm{C}_{9} \mathrm{H}_{6} \mathrm{O}_{3}\right]^{-}$, $\left[\mathrm{M}-\mathrm{H}-\mathrm{C}_{7} \mathrm{H}_{10} \mathrm{O}_{5}\right]^{-}$, [M$\left.\mathrm{H}-\mathrm{C}_{9} \mathrm{H}_{6} \mathrm{O}_{3}-\mathrm{H}_{2} \mathrm{O}\right]^{-}$, [M- $\left.\mathrm{M}-\mathrm{C}_{7} \mathrm{H}_{10} \mathrm{O}_{5}-\mathrm{H}_{2} \mathrm{O}\right]^{-}$, [M-H- $\left.\mathrm{C}_{9} \mathrm{H}_{6} \mathrm{O}_{3}-2 \mathrm{H}_{2} \mathrm{O}\right]^{-}$, [M- $\left.\mathrm{M}-\mathrm{C}_{9} \mathrm{H}_{6} \mathrm{O}_{3}-3 \mathrm{H}_{2} \mathrm{O}\right]^{-}$, [M-H-C $\left.\mathrm{H}_{70} \mathrm{H}_{10} \mathrm{O}_{5}-\mathrm{CO}_{2}\right]^{-}$and $\left[\mathrm{M}-\mathrm{H}-\mathrm{C}_{9} \mathrm{H}_{6} \mathrm{O}_{3}-3 \mathrm{H}_{2} \mathrm{O}-\mathrm{CO}_{2}\right]^{-}$. Figure S3-B has shown the main cracking pathways of chlorogenic acid. Compounds $16,32,33,77$ were filtered by $\mathrm{m} / z 161.02 \mathrm{Da}$ and $135.04 \mathrm{Da}$, which were an indicative of caffeic acid derivatives. Take compound $77\left(\mathrm{C}_{11} \mathrm{H}_{12} \mathrm{O}_{4}\right)$ as an example, the [M-H] ${ }^{-}$at $m / z 207.0662$, and the secondary mass spectrometry were detected at $m / z$ $179.0341[\mathrm{M}-\mathrm{H}-\mathrm{CO}]^{-}, \mathrm{m} / \mathrm{z} 161.0234\left[\mathrm{M}-\mathrm{H}-\mathrm{CO}-\mathrm{H}_{2} \mathrm{O}\right]^{-}, \mathrm{m} / z 135.0440\left[\mathrm{M}-\mathrm{H}-\mathrm{CO}_{-} \mathrm{CO}_{2}\right]^{-}$, which tentatively identified as ethyl caffeate. Compounds 32 and $\mathbf{3 3}$ also have a similar pyrolysis law.

\section{Identification of iridoids}

The most basic core of iridoids is iridoid alcohol, containing cyclic enethers and alcoholic hydroxyl groups, which imply the basic skeleton of iridoid glycosides contains a characteristic dihydropyran ring is cis connected to a cyclopentane unit structure. A total of 15 iridoids were detected [M-H] ${ }^{-}$in negative ion mode. In the $\mathrm{ESI}^{-}$mode, the fragment ion ${ }^{2,7} \mathrm{FO}^{-}$ion at $\mathrm{m} / \mathrm{z} 101.02$ obtained by the fragmentation of the aglycon part of the excimer ion, which was a characteristic ion to identify the structure of the excimer ion [25, 26]. According to the literature [27], the ion at $\mathrm{m} / \mathrm{z} 147.03$ was the prominent ion of iridoids. Compounds 5 , $8,11,13$ and 40 were detected by characteristic ions $147.03 \mathrm{Da}$ or $101.02 \mathrm{Da}$. Taking the derivation process of compound 8 as an example, the quasimolecular ion peak of compound 8 was $m / z 389.1089[\mathrm{M}-\mathrm{H}]^{-}$, yielded a formula of $\mathrm{C}_{16} \mathrm{H}_{22} \mathrm{O}_{11}$. The $[\mathrm{M}-\mathrm{H}]^{-}$ion of $\mathrm{m} / z 227.0550$ was the absence of glucose neutral fragment from $\mathrm{m} / \mathrm{z}$ 389.1089. The fragment ions $\mathrm{m} / \mathrm{z}$ of 209.0356 and 183.0655 were losing one molecule of $\mathrm{H}_{2} \mathrm{O}$ and one molecule of $\mathrm{CO}_{2}$ from [M$\mathrm{H}]^{-}$ion of $\mathrm{m} / \mathrm{z} 227.0550$. Then the $[\mathrm{M}-\mathrm{H}]^{-}$ion of $\mathrm{m} / \mathrm{z} 183.0655$ continuous loss two molecule of $\mathrm{H}_{2} \mathrm{O}$, convert to the fragment ions of $\mathrm{m} / z 165.0543$ and $m / z$ 147.0285. Consistently, the dehydration of fragment ion $\mathrm{m} / \mathrm{z} 209.0356$ leads to the production of $\mathrm{m} / \mathrm{z} 191.0553$. And the fragment ion $\mathrm{m} / \mathrm{z} 147.0285 \mathrm{was}$ decarboxylation of $m / z$ 191.0553. The cleavage detail of each ion was displayed in Figure S3-C.

In addition, iridoid glycosides are usually connected to a glucose at the $\mathrm{C} 1$ position, so they are easy to lose neutral fragments such as $162.02 \mathrm{Da}$ (glc), 44.01 $\mathrm{Da}\left(\mathrm{CO}_{2}\right)$ and $18.01 \mathrm{Da}\left(\mathrm{H}_{2} \mathrm{O}\right)$ [28]. A total of five compounds $(7,10,21,28,48)$ were filtered by $162.02 \mathrm{Da}$. Compound $7\left(\mathrm{C}_{16} \mathrm{H}_{22} \mathrm{O}_{10}\right)$ showed the $[\mathrm{M}-\mathrm{H}]{ }^{-}$at $m / z$ 373.1140, the fragment ions determined from MS/MS spectra were $m / z 211.0606[\mathrm{M}-\mathrm{H}-\mathrm{glc}]^{-}, \mathrm{m} / z 193.0498\left[\mathrm{M}-\mathrm{H}-\mathrm{glc}-\mathrm{H}_{2} \mathrm{O}\right]^{-}, m / z 167.0703\left[\mathrm{M}-\mathrm{H}-\mathrm{glc}-\mathrm{CO}_{2}\right]$, and $\mathrm{m} / z 149.0598$ [M-H-glc- $\mathrm{CO}_{2}-\mathrm{H}_{2} \mathrm{O}$ ], $\mathrm{m} / z 211.0940$ (373.1140 Da - 162.02 Da) were characteristic fragments of compound 7, which tentatively identified as geniposidic acid. To sum up, the iridoids were easier to lose the glucose neutral fragment ion $162.02 \mathrm{Da}$, and obtain aglycon fragment ions, and then the aglycon ions decarboxylated or dehydrated to become a series of fragments.

\section{Identification of lignans}


A large number of the bisepoxylignans and monoepoxylignans combine with glucose to form monoglycoside or diglycoside. Therefore, the majority of them could lose glycosyl and methyl neutral fragments first, then lose one or two molecular of $\mathrm{CH}_{2} \mathrm{O}$, and finally formed $151.03 \mathrm{Da}$. Therefore, characteristic ion fragment 151.03 Da was uesd to identify lignans. Compound $\mathbf{3 1}, \mathbf{4 4}, \mathbf{5 2}, \mathbf{5 3}, 101$ and 132 were detected by FSCIF with 151.03 Da. Compound $\mathbf{4 4}$ showed the $[\mathrm{M}-\mathrm{H}]^{-}$at $m / z$ 681.2400, the fragment ions $\mathrm{m} / \mathrm{z}$ 519.5070, $\mathrm{m} / \mathrm{z} 357.1346, \mathrm{~m} / \mathrm{z}$ 151.0390, which were corresponding to [M-H-glc] ${ }^{-}$, [M-H-glc-glc] ${ }^{-}$and [M-H-glc- $^{-}$ glc- $\left.\mathrm{C}_{12} \mathrm{H}_{14} \mathrm{O}_{3}\right]^{-}$. Subsequncely, compound 37, 39, 138 and 139 filtered by NLF with ions of 162.02 Da, 44.01 Da or 18.01 Da.

\section{Other compounds}

A total of 11 polyphenol $(\mathbf{3}, \mathbf{6}, \mathbf{9}, \mathbf{1 7}, \mathbf{1 8}, \mathbf{2 0}, \mathbf{2 3}, \mathbf{2 4}, \mathbf{2 5}, \mathbf{3 4}, \mathbf{3 5})$ were recognized by FSNLF analysis. Because of the presence of hydroxyl and carboxyl groups, these compounds were filtered by $18.01 \mathrm{Da}\left(\mathrm{H}_{2} \mathrm{O}\right)$ and $44.01 \mathrm{Da}\left(\mathrm{CO}_{2}\right)$. In addition, six other compounds were identified by comparison with the literature.

\section{Assay Validation}

\section{Quantification of 19 major compounds in MTBD}

The 19 compounds quantified were the screening of osteoporosis targets by network pharmacology in the early stage of our laboratory, then the representative and top ranked compounds were selected. Methodology analysis showed that the assay method of 19 compounds (including three pairs of isomers) had a good repeatability and stability.

\section{Specificity.}

The chromatograms of blank sample, standard mixture sample, and MTBD extracts sample were presented in Fig. 4. Nineteen compounds in MTBD extracts were separated within 25 minutes, which baseline separation of each compound was achieved and no obvious signal noises were occurred around determinate peak. Additionally, no interferences were detected between the three isomers.

\section{Linearity and lower limit of quantification.}

Three batches of standard curve solutions with six different concentrations were prepared. The typical standard curves were assessed by using DAS 2.0 software with the quadratic weight $\left(\mathrm{W}=1 / \mathrm{C}^{2}\right)$. The standard curves, LLOQ and correlation coefficients were listed in Table 2, proved that the calibration curves of the components with a good linearity over the studied concentration range.

Table 2

Calibration curves, linear range, $r^{2}$ and LOQs of 19 compounds in MTBD

\begin{tabular}{|lllll|}
\hline Compound & Calibration curves & $\begin{array}{l}\text { Linear range } \\
(\mu \mathrm{g} / \mathrm{mL})\end{array}$ & $r^{2}$ & $\begin{array}{l}\text { LLOQ } \\
(\mu \mathrm{g} / \mathrm{mL})\end{array}$ \\
\hline ICGAA & $y=647.937 x+1.476$ & $4.800-192.000$ & 0.9994 & 4.800 \\
\hline 1,5-DQA & $y=364.018 x-0.810$ & $6.100-244.000$ & 0.9991 & 6.810 \\
\hline GE & $y=22386.297 x-0.191$ & $0.023-0.920$ & 0.9982 & 0.025 \\
\hline APG & $y=29960.140 x-0.562$ & $0.065-2.600$ & 0.9989 & 0.073 \\
\hline LT & $y=15454.517 x-0.090$ & $0.015-0.600$ & 0.9986 & 0.015 \\
\hline KPF & $y=9208.139 x-0.051$ & $0.010-0.400$ & 0.9983 & 0.010 \\
\hline QC & $y=8437.051 x-0.062$ & $0.011-0.440$ & 0.9989 & 0.011 \\
\hline A-7-O-G & $y=6901.196 x+11.968$ & $4.200-168.000$ & 0.9962 & 4.200 \\
\hline RU & $y=2560.803 x-0.093$ & $0.230-9.200$ & 0.9992 & 0.250 \\
\hline HSYA & $y=1104.734 x-0.935$ & $9.830-392.000$ & 0.9984 & 10.930 \\
\hline NG-R 1 & $y=8.300 x-0.016$ & $2.400-96.000$ & 0.9964 & 2.400 \\
\hline G-Re & $y=730.821 x+0.137$ & $1.010-40.400$ & 0.9963 & 1.010 \\
\hline G-Rg ${ }_{1}$ & $y=21.700 x+0.029$ & $6.500-260.000$ & 0.9983 & 6.700 \\
\hline G-Rb ${ }_{1}$ & $y=93.320 x+0.128$ & $5.660-226.400$ & 0.9979 & 5.830 \\
\hline CA & $y=183840.263 x-8.999$ & $0.260-10.400$ & 0.9985 & 0.260 \\
\hline FA & $y=2322.874 x-0.525$ & $0.390-15.600$ & 0.9992 & 0.410 \\
\hline GPA & $y=119.544 x-0.011$ & $0.800-32.000$ & 0.9987 & 0.800 \\
\hline CGA & $y=3414.355 x-5.245$ & $5.500-220.000$ & 0.9978 & 5.660 \\
\hline PDG & $y=10.556 x-0.018$ & $2.180-87.200$ & 0.9987 & 2.180 \\
\hline
\end{tabular}

Page $16 / 25$ 
Precision and accuracy.

Three batches of quality control samples were prepared according to three concentration levels. Each concentration was analyzed with 6 duplications. The precision values were between $1.13 \% \sim 6.66 \%$ for intra-day, $2.42 \% \sim 10.62 \%$ for inter-day and accuracy was range from $86.11-114.27 \%$. The results above demonstrated the acceptable precision and accuracy of the present method.

Repeatability and stability.

Six MTBD sample extracts were prepared on the same day, the repeatability of 19 components was within $6.26 \%$ relative standard deviation (RSD). The stability results of 19 compounds were summarized in Table 3, the acceptability of the data within $3.92 \%$ deviation from the $0 \mathrm{~h}$ sample values, which indicated that a large number of samples could be stable in each analytical run.

Table 3

Precision, repeatability, stability and recovey of 19 compounds in MTBD

\begin{tabular}{|c|c|c|c|c|c|c|c|c|c|c|}
\hline \multirow[t]{2}{*}{ Compound } & \multicolumn{3}{|c|}{$\begin{array}{l}\text { Inter-day precision } \\
(R S D, \mathrm{n}=3)\end{array}$} & \multicolumn{3}{|c|}{$\begin{array}{l}\text { Intra-day precision } \\
(R S D, \mathrm{n}=3)\end{array}$} & \multirow{2}{*}{$\begin{array}{l}\text { Repeatability } \\
\text { (RSD, n = } \\
6, \%)\end{array}$} & \multirow{2}{*}{$\begin{array}{l}\text { Stability } \\
\text { (RSD, } n=4, \%)\end{array}$} & \multicolumn{2}{|l|}{$\begin{array}{l}\text { Accuracy } \\
(n=6, \%)\end{array}$} \\
\hline & Low & Middle & High & Low & Middle & High & & & Recovey & RSD \\
\hline ICGAA & 4.74 & 5.61 & 6.45 & 1.24 & 2.34 & 3.39 & 2.62 & 3.13 & 107.36 & 1.76 \\
\hline 1,5-DQA & 3.37 & 4.31 & 5.18 & 1.13 & 0.59 & 1.63 & 6.26 & 3.48 & 106.46 & 2.11 \\
\hline GE & 2.42 & 7.24 & 4.87 & 2.29 & 2.79 & 0.90 & 2.30 & 2.68 & 106.41 & 2.06 \\
\hline APG & 3.34 & 5.34 & 3.64 & 3.22 & 2.58 & 2.82 & 1.70 & 2.18 & 106.73 & 2.23 \\
\hline LT & 4.05 & 7.84 & 4.53 & 3.13 & 2.81 & 1.09 & 4.75 & 3.92 & 98.21 & 3.01 \\
\hline KPF & 9.47 & 9.00 & 7.98 & 2.18 & 1.54 & 5.51 & 4.94 & 3.60 & 103.12 & 2.37 \\
\hline $\mathrm{QC}$ & 8.67 & 6.94 & 5.52 & 6.66 & 2.25 & 1.35 & 4.68 & 2.81 & 103.42 & 4.10 \\
\hline A-7-0-G & 4.17 & 3.42 & 7.38 & 4.15 & 3.05 & 3.13 & 2.09 & 2.38 & 104.56 & 1.87 \\
\hline $\mathrm{RU}$ & 4.03 & 3.23 & 3.42 & 1.86 & 1.92 & 2.57 & 2.99 & 2.34 & 105.39 & 0.92 \\
\hline HSYA & 3.81 & 4.97 & 5.67 & 2.25 & 1.13 & 4.78 & 3.40 & 2.56 & 104.80 & 1.13 \\
\hline NG-R1 & 8.22 & 4.19 & 5.85 & 1.63 & 2.07 & 4.55 & 4.05 & 0.75 & 104.87 & 2.81 \\
\hline G-RE & 6.50 & 3.92 & 5.41 & 3.17 & 1.38 & 2.47 & 4.02 & 3.23 & 107.35 & 1.73 \\
\hline G-RG1 & 6.10 & 7.59 & 6.12 & 5.08 & 4.64 & 4.80 & 3.27 & 2.26 & 104.64 & 1.59 \\
\hline G-RB1 & 10.62 & 3.37 & 3.61 & 2.94 & 2.71 & 3.47 & 4.50 & 2.99 & 106.94 & 2.54 \\
\hline $\mathrm{CA}$ & 6.29 & 5.83 & 4.08 & 5.45 & 3.62 & 4.31 & 2.15 & 2.43 & 101.10 & 0.53 \\
\hline FA & 8.76 & 7.21 & 7.61 & 3.90 & 4.15 & 2.40 & 2.94 & 3.74 & 105.71 & 2.10 \\
\hline GPA & 8.36 & 6.89 & 10.52 & 2.64 & 0.54 & 4.11 & 3.82 & 3.59 & 107.60 & 3.01 \\
\hline CGA & 2.74 & 5.12 & 2.82 & 2.52 & 1.25 & 3.50 & 2.99 & 2.24 & 106.59 & 2.11 \\
\hline PDG & 6.95 & 7.32 & 7.71 & 2.69 & 2.78 & 4.11 & 2.67 & 1.97 & 92.08 & 4.57 \\
\hline
\end{tabular}

\section{Application to samples Modified Tabusen-2 decoction (MTBD)}

The method established above was successfully utilized to quantitative studies of MTBD extracts, as shown in Table 4. Eight batches of MTBD samples prepared with different herb sources were determined by using above mature method. The herb formulation of each batch was listed in Table $\mathbf{S 1}$. There is an indicative of that the concentrations of 19 compounds varied significantly in MTBD extracts, the content of flavonoids was the highest, followed by saponins (Figure S4), which attracted the attention of herb quality in picking as well as in circulating during the market. We should get the hints from the result of MTBD extracts quantitation research that strictly controlled the herb quality need to pay highly attention, which was a guarantee of their clinical efficacy and safety. 
Table 4

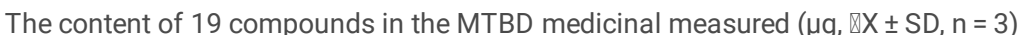

\begin{tabular}{|c|c|c|c|c|c|c|c|c|c|c|c|c|c|c|}
\hline Compound & Batch 1 & & Batch 2 & & Batch 3 & & Batch 4 & & Batch 5 & & Batch 6 & & Batch 7 & \\
\hline & $\begin{array}{l}\text { Content } \\
(\mu \mathrm{g})\end{array}$ & $\begin{array}{l}\text { RSD } \\
(\%)\end{array}$ & $\begin{array}{l}\text { Content } \\
(\mu \mathrm{g})\end{array}$ & $\begin{array}{l}\text { RSD } \\
(\%)\end{array}$ & $\begin{array}{l}\text { Content } \\
(\mu \mathrm{g})\end{array}$ & $\begin{array}{l}\text { RSD } \\
(\%)\end{array}$ & $\begin{array}{l}\text { Content } \\
(\mu \mathrm{g})\end{array}$ & $\begin{array}{l}\text { RSD } \\
(\%)\end{array}$ & $\begin{array}{l}\text { Content } \\
(\mu \mathrm{g})\end{array}$ & $\begin{array}{l}\text { RSD } \\
(\%)\end{array}$ & $\begin{array}{l}\text { Content } \\
(\mu \mathrm{g})\end{array}$ & $\begin{array}{l}\text { RSD } \\
(\%)\end{array}$ & $\begin{array}{l}\text { Content } \\
(\mu \mathrm{g})\end{array}$ & $\begin{array}{l}1 \\
1\end{array}$ \\
\hline ICGAA & $\begin{array}{l}16762.18 \\
\pm \\
199.87\end{array}$ & 1.19 & $\begin{array}{l}11295.32 \pm \\
149.83\end{array}$ & 1.33 & $\begin{array}{l}13711.97 \\
\pm \\
574.45\end{array}$ & 4.19 & $\begin{array}{l}14877.31 \\
\pm \\
411.70\end{array}$ & 2.77 & $\begin{array}{l}18213.17 \\
\pm \\
604.40\end{array}$ & 3.32 & $\begin{array}{l}17164.28 \pm \\
553.79\end{array}$ & 3.23 & $\begin{array}{l}16781.86 \pm \\
743.30\end{array}$ & ? \\
\hline 1,5-DQA & $\begin{array}{l}13869.07 \\
\pm \\
653.18\end{array}$ & 4.71 & $\begin{array}{l}12125.54 \pm \\
324.70\end{array}$ & 2.68 & $\begin{array}{l}3151.23 \\
\pm \\
42.20\end{array}$ & 1.34 & $\begin{array}{l}9869.47 \pm \\
186.95\end{array}$ & 1.89 & $\begin{array}{l}14198.79 \\
\pm \\
468.05\end{array}$ & 3.30 & $\begin{array}{l}15605.54 \pm \\
768.51\end{array}$ & 4.92 & $\begin{array}{l}5106.09 \pm \\
189.07\end{array}$ & $\vdots$ \\
\hline GE & $\begin{array}{l}21.46 \pm \\
1.26\end{array}$ & 4.73 & $\begin{array}{l}12.26 \pm \\
0.03\end{array}$ & 0.21 & $\begin{array}{l}7.16 \pm \\
0.17\end{array}$ & 2.35 & $\begin{array}{l}29.67 \pm \\
0.49\end{array}$ & 1.67 & $\begin{array}{l}24.35 \pm \\
1.46\end{array}$ & 6.03 & $\begin{array}{l}18.19 \pm \\
0.41\end{array}$ & 2.27 & $\begin{array}{l}11.35 \pm \\
0.46\end{array}$ & 4 \\
\hline APG & $\begin{array}{l}193.44 \pm \\
7.91\end{array}$ & 4.09 & $\begin{array}{l}30.00 \pm \\
0.51\end{array}$ & 1.73 & $\begin{array}{l}180.55 \pm \\
7.09\end{array}$ & 3.93 & $\begin{array}{l}61.60 \pm \\
2.16\end{array}$ & 3.52 & $\begin{array}{l}173.06 \pm \\
9.52\end{array}$ & 5.50 & $\begin{array}{l}26.22 \pm \\
0.97\end{array}$ & 3.73 & $\begin{array}{l}221.23 \pm \\
5.14\end{array}$ & ; \\
\hline LT & $\begin{array}{l}27.43 \pm \\
1.31\end{array}$ & 4.80 & $\begin{array}{l}9.46 \pm \\
0.46\end{array}$ & 4.87 & $\begin{array}{l}28.72 \pm \\
1.06\end{array}$ & 3.70 & $\begin{array}{l}11 . .38 \pm \\
0.49\end{array}$ & 4.32 & $\begin{array}{l}24.46 \pm \\
0.85\end{array}$ & 3.49 & $\begin{array}{l}6.33 \pm \\
0.36\end{array}$ & 5.74 & $\begin{array}{l}38.02 \pm \\
1.77\end{array}$ & 4 \\
\hline $\mathrm{KPF}$ & $\begin{array}{l}8.76 \pm \\
0.18\end{array}$ & 2.13 & $\begin{array}{l}5.67 \pm \\
0.10\end{array}$ & 1.90 & $\begin{array}{l}7.52 \pm \\
0.22\end{array}$ & 2.96 & $\begin{array}{l}5.84 \pm \\
0.27\end{array}$ & 4.76 & $\begin{array}{l}11.25 \pm \\
0.28\end{array}$ & 2.46 & $\begin{array}{l}7.14 \pm \\
0.24\end{array}$ & 3.31 & $\begin{array}{l}15.15 \pm \\
0.86\end{array}$ & ! \\
\hline $\mathrm{QC}$ & $\begin{array}{l}5.88 \pm \\
0.04\end{array}$ & 0.64 & $\begin{array}{l}3.89 \pm \\
0.06\end{array}$ & 1.46 & $\begin{array}{l}5.65 \pm \\
0.29\end{array}$ & 5.15 & $\begin{array}{l}4.32 \pm \\
0.16\end{array}$ & 3.77 & $\begin{array}{l}8.00 \pm \\
0.32\end{array}$ & 4.03 & $\begin{array}{l}4.29 \pm \\
0.21\end{array}$ & 4.79 & $\begin{array}{l}9.29 \pm \\
0.26\end{array}$ & ; \\
\hline A-7-O-G & $\begin{array}{l}1338.14 \\
\pm \\
56.00\end{array}$ & 4.18 & $\begin{array}{l}522.55 \pm \\
12.41\end{array}$ & 2.37 & $\begin{array}{l}2642.11 \\
\pm \\
111.36\end{array}$ & 4.21 & $\begin{array}{l}1005.98 \\
\pm \\
28.87\end{array}$ & 2.87 & $\begin{array}{l}1485.53 \\
\pm \\
73.13\end{array}$ & 4.92 & $\begin{array}{l}418.74 \pm \\
15.29\end{array}$ & 3.65 & $\begin{array}{l}3759.35 \pm \\
132.56\end{array}$ & $\vdots$ \\
\hline $\mathrm{RU}$ & $\begin{array}{l}278.13 \pm \\
11.11\end{array}$ & 4.00 & $\begin{array}{l}249.04 \pm \\
5.18\end{array}$ & 2.08 & $\begin{array}{l}224.49 \pm \\
5.36\end{array}$ & 2.39 & $\begin{array}{l}272.52 \pm \\
8.27\end{array}$ & 3.03 & $\begin{array}{l}299.01 \pm \\
9.27\end{array}$ & 3.10 & $\begin{array}{l}405.94 \pm \\
10.02\end{array}$ & 2.47 & $\begin{array}{l}353.85 \pm \\
6.71\end{array}$ & 1 \\
\hline HSYA & $\begin{array}{l}36875.52 \\
\pm \\
584.45\end{array}$ & 1.58 & $\begin{array}{l}32683.88 \pm \\
1097.05\end{array}$ & 3.36 & $\begin{array}{l}32605.85 \\
\pm \\
1720.72\end{array}$ & 5.28 & $\begin{array}{l}36899.09 \\
\pm \\
1252.44\end{array}$ & 3.39 & $\begin{array}{l}36566.99 \\
\pm \\
1173.24\end{array}$ & 3.21 & $\begin{array}{l}37101.52 \pm \\
865.43\end{array}$ & 2.33 & $\begin{array}{l}35625.68 \pm \\
1346.05\end{array}$ & $\vdots$ \\
\hline NG-R1 & $\begin{array}{l}2286.91 \\
\pm \\
110.69\end{array}$ & 4.84 & $\begin{array}{l}1761.26 \pm \\
79.66\end{array}$ & 4.52 & $\begin{array}{l}2534.44 \\
\pm \\
68.48\end{array}$ & 2.70 & $\begin{array}{l}2245.17 \\
\pm \\
104.11\end{array}$ & 4.64 & $\begin{array}{l}3040.46 \\
\pm \\
147.89\end{array}$ & 4.86 & $\begin{array}{l}2719.07 \pm \\
86.30\end{array}$ & 3.17 & $\begin{array}{l}3063.47 \pm \\
91.59\end{array}$ & ; \\
\hline G-Re & $\begin{array}{l}115.34 \pm \\
2.04\end{array}$ & 1.18 & $\begin{array}{l}173.72 \pm \\
2.04\end{array}$ & 1.18 & $\begin{array}{l}138.15 \pm \\
3.66\end{array}$ & 2.65 & $\begin{array}{l}128.01 \pm \\
2.95\end{array}$ & 2.30 & $\begin{array}{l}129.25 \pm \\
3.70\end{array}$ & 2.86 & $\begin{array}{l}154.83 \pm \\
4.72\end{array}$ & 3.05 & $\begin{array}{l}117.29 \pm \\
1.20\end{array}$ & 1 \\
\hline G-Rg1 & $\begin{array}{l}16762.12 \\
\pm \\
199.87\end{array}$ & 1.19 & $\begin{array}{l}21295.33 \pm \\
149.83\end{array}$ & 1.33 & $\begin{array}{l}13711.97 \\
\pm \\
574.45\end{array}$ & 4.19 & $\begin{array}{l}14877.31 \\
\pm \\
411.70\end{array}$ & 2.77 & $\begin{array}{l}18213.17 \\
\pm \\
604.40\end{array}$ & 3.32 & $\begin{array}{l}17164.28 \pm \\
553.80\end{array}$ & 3.23 & $\begin{array}{l}18534.92 \pm \\
541.17\end{array}$ & 2 \\
\hline G-Rb1 & $\begin{array}{l}8989.89 \\
\pm \\
161.19\end{array}$ & 1.79 & $\begin{array}{l}8170.53 \pm \\
276.59\end{array}$ & 3.39 & $\begin{array}{l}8514.05 \\
\pm \\
329.66\end{array}$ & 3.87 & $\begin{array}{l}8002.95 \\
\pm \\
114.29\end{array}$ & 1.43 & $\begin{array}{l}10322.41 \\
\pm \\
184.74\end{array}$ & 1.79 & $\begin{array}{l}11080.66 \pm \\
554.08\end{array}$ & 5.00 & $\begin{array}{l}9488.45 \pm \\
406.75\end{array}$ & 2 \\
\hline $\mathrm{CA}$ & $\begin{array}{l}684.33 \pm \\
0.99\end{array}$ & 0.14 & $\begin{array}{l}555.93 \pm \\
19.10\end{array}$ & 3.44 & $\begin{array}{l}980.54 \pm \\
29.49\end{array}$ & 3.01 & $\begin{array}{l}362.15 \pm \\
16.41\end{array}$ & 4.53 & $\begin{array}{l}601.07 \pm \\
11.68\end{array}$ & 1.94 & $\begin{array}{l}489.06 \pm \\
13.84\end{array}$ & 2.83 & $\begin{array}{l}953.46 \pm \\
25.03\end{array}$ & ; \\
\hline FA & $\begin{array}{l}142.20 \pm \\
2.60\end{array}$ & 1.83 & $\begin{array}{l}208.53 \pm \\
6.40\end{array}$ & 3.07 & $\begin{array}{l}211.98 \pm \\
7.80\end{array}$ & 3.68 & $\begin{array}{l}211.14 \pm \\
9.22\end{array}$ & 4.17 & $\begin{array}{l}271.42 \pm \\
1.19\end{array}$ & 0.44 & $\begin{array}{l}468.62 \pm \\
8.42\end{array}$ & 1.80 & $\begin{array}{l}532.06 \pm \\
12.35\end{array}$ & ; \\
\hline GPA & $\begin{array}{l}3146.68 \\
\pm \\
108.83\end{array}$ & 3.46 & $\begin{array}{l}3163.53 \pm \\
123.27\end{array}$ & 3.90 & $\begin{array}{l}3112.44 \\
\pm \\
116.01\end{array}$ & 3.73 & $\begin{array}{l}3045.59 \\
\pm \\
86.91\end{array}$ & 2.85 & $\begin{array}{l}3163.44 \\
\pm \\
41.17\end{array}$ & 1.30 & $\begin{array}{l}3284.01 \pm \\
73.66\end{array}$ & 2.24 & $\begin{array}{l}3173.78 \pm \\
118.37\end{array}$ & $\vdots$ \\
\hline CGA & $\begin{array}{l}11069.83 \\
\pm \\
373.70\end{array}$ & 3.38 & $\begin{array}{l}21193.91 \pm \\
16.47\end{array}$ & 0.08 & $\begin{array}{l}6736.66 \\
\pm \\
230.72\end{array}$ & 3.42 & $\begin{array}{l}11708.75 \\
\pm \\
230.42\end{array}$ & 1.97 & $\begin{array}{l}11213.11 \\
\pm \\
322.76\end{array}$ & 2.88 & $\begin{array}{l}20884.47 \pm \\
710.15\end{array}$ & 3.40 & $\begin{array}{l}8027.72 \pm \\
378.50\end{array}$ & 2 \\
\hline
\end{tabular}

Page 18/ 25 


\begin{tabular}{|c|c|c|c|c|c|c|c|c|c|c|c|c|c|c|}
\hline \multirow[t]{2}{*}{ Compound } & \multicolumn{2}{|l|}{ Batch 1} & \multicolumn{2}{|l|}{ Batch 2} & \multicolumn{2}{|l|}{ Batch 3} & \multicolumn{2}{|l|}{ Batch 4} & \multicolumn{2}{|l|}{ Batch 5} & \multicolumn{2}{|l|}{ Batch 6} & \multicolumn{2}{|l|}{ Batch 7} \\
\hline & $\begin{array}{l}\text { Content } \\
(\mu g)\end{array}$ & $\begin{array}{l}\text { RSD } \\
(\%)\end{array}$ & $\begin{array}{l}\text { Content } \\
(\mu \mathrm{g})\end{array}$ & $\begin{array}{l}\text { RSD } \\
(\%)\end{array}$ & $\begin{array}{l}\text { Content } \\
(\mu \mathrm{g})\end{array}$ & $\begin{array}{l}\text { RSD } \\
(\%)\end{array}$ & $\begin{array}{l}\text { Content } \\
(\mu \mathrm{g})\end{array}$ & $\begin{array}{l}\text { RSD } \\
\text { (\%) }\end{array}$ & $\begin{array}{l}\text { Content } \\
(\mu g)\end{array}$ & $\begin{array}{l}\text { RSD } \\
(\%)\end{array}$ & $\begin{array}{l}\text { Content } \\
(\mu g)\end{array}$ & $\begin{array}{l}\text { RSD } \\
\text { (\%) }\end{array}$ & $\begin{array}{l}\text { Content } \\
(\mu g)\end{array}$ & $\begin{array}{l}1 \\
1\end{array}$ \\
\hline PDG & $\begin{array}{l}8372.22 \\
\pm \\
65.92\end{array}$ & 0.79 & $\begin{array}{l}7228.23 \pm \\
224.48\end{array}$ & 3.11 & $\begin{array}{l}8303.93 \\
\pm \\
75.14\end{array}$ & 0.90 & $\begin{array}{l}8145.20 \\
\pm \\
244.72\end{array}$ & 3.00 & $\begin{array}{l}7812.40 \\
\pm \\
325.53\end{array}$ & 4.17 & $\begin{array}{l}6784.62 \pm \\
257.26\end{array}$ & 3.79 & $\begin{array}{l}8551.33 \pm \\
250.91\end{array}$ & i \\
\hline
\end{tabular}

\section{Discussion}

Although the isolation and purification before biological activity evaluation is a traditional strategy of exploring material basis in TMM, the time-consuming and labor intensive characteristics cannot be neglected. Ultraviolet (UV) detector is exceedingly common for flavanoids, phenylpropanines, and other UVabsorbing compounds [29], while is not applicable to the analysis of saponin. Although the detection of saponin could be enabled by evaporative light scattering detector (ELSD), the sensitivity during the test procedure should also be aware of [30]. Herein, in order to shorten the analysis time, improve the analysis sensitivity, and simultaneously determine UV- absorbing compounds and Non-UV-absorbing compounds, high performance liquid chromatography coupled with mass spectrometry (HPLC-QE Orbitrap MS) approach [31], as a high efficiency, FSCIF and sensitive technology, is employed in this study to simultaneouses isolate and identify the material basis in MTBD. Additionally, the existence of isomer (ICGAA with 1, 5-DQA, GE with APG, LT with KPF) in MTBD increases the difficulty of separation and analysis [32]. The chromatographic conditions in quantitative analysis need to be optimized carefully during present research.

In order to achieve better separation effect for three pairs of isomers in MTBD, the mobile phase was screened in this experiment. The peak of each component was with symmetrical shape and no tailing phenomenon. Additionally, the influence of column temperature and flow rate was considered, and a better separation was achieved under column temperature of $30^{\circ} \mathrm{C}$ and flow rate of $0.3 \mathrm{~mL} / \mathrm{min}$. A variety of chromatographic columns were also optimized in

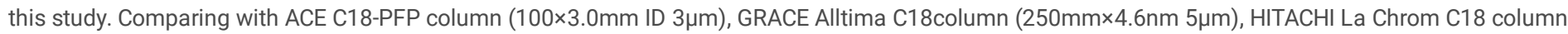
$(250 \mathrm{~mm} \times 4.6 \mathrm{~mm}$ I.D. $5 \mu \mathrm{m})$, Thermo ODS-2 HYPERSIL column $(250 \mathrm{~mm} \times 4.6 \mathrm{~mm}, 5 \mu \mathrm{m})$, ACE C18-PFP column performed better separation and resolution, especially for the three isomers.

It was found through analysis that the contents of the 19 components differ in MTBD prepared from different batches of crude drugs, this might be because the crude drugs of different batches were different in origin, growing environments. This has aroused our attention in all aspects of picking, transportation.

\section{Conclusions}

Based on HPLC-QE Orbitrap MS with FSCIF approach to rapid detect structure fragment and quantification of major representative components in MTBD, 143 compounds with seven chemical categories were unambiguously or tentatively identified. This study not only enriched the cleavage law of MTBD compounds, but also established a approach for the accurate search and discovery of active components from complex mixtures. The repeatability, accuracy, stability, linearity, recoveries, and reproducibility of quantitative analysis all meet the criteria for acceptability of quantitative studies. The determination of 19 compounds in MTBD extracts in different batches were obtained to monitor the quality of each prescription, which facilitates the better development of quality evaluation technique in MTBD and will help to further exploration of quality control of MTBD.The 19 compounds determined based on the qualitative and quantitative results are the major components of the MTDB, this work promotes the development and utilization of traditional prescriptions and lays the material foundation for their pharmacokinetics.

\section{Abbreviations}

MTBD: Modified Tabusen-2 decoction; TBD: Tabusen-2 decoction; EU: Eucommia ulmoides Oliver;EC: Echinops latifolius Tausch; CT: Carthamus tinctorius L; PN: Panax Notoginseng, TCM: Traditional Chinese Medicine; TMM: Traditional Mongolian Medicine; ICGAA: Isochlorogenic acid A; 1,5-DQA: 1,5Dicaffeoylqunic acid; GE: Genistein; APG: Apigenin; LT: Luteolin; KPF: Kaempferol; QC: Quercetin; A-7-0-G: Apigenin-7-0-glucuronide; RT: Rutin; HSYA:

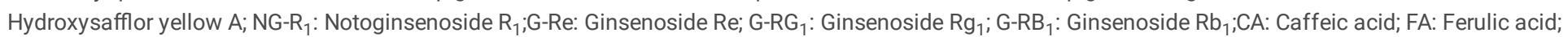
GPA: Geniposidic acid; CGA: Chlorogenic acid; PDG: Pinoresinol diglucoside; IS: Internal standard.

\section{Declarations}

Acknowledgements: Not applicable.

Funding: This work was financially supported by National Natural Science Foundation of China (81860756, 81960758), Innovation Guide Project (02039001), and Inner Mongolia Autonomous Region Higher Education Science Research Project (NJZY19099).

Competing interests: The authors declare that there are no conflicts of interest.

Availability of data and material:All the data involved in this experiment is true and valid. 
Author contributions:All authors contributed to the study conception and design. P F X and B Q conceived and designed the study; $Y Z$ and $X \mathrm{D}$ performed the experiment and prepared the manuscript; Q X F analyzed the data, R D and R B graphed the picture.

Author details: ${ }^{1}$ School of Inner Mongolia Medical University, Jinshan development zone, Hohhot,010110, China.

All authors read and approved the final manuscript.

Availability of data and materials: The research data generated from this study is included within the article.

Ethics approval and consent to participate: Not applicable.

Consent for publication: Not applicable.

Compliance with Ethical Standards:This article does not contain any studies with human participants or animals performed by any of the authors.

\section{References}

1. Xie J, Tan Z, Guo Y, Yang H, Hu G. Quality standard control of Modified Tabusen-2 decoction and TLC identification method of Eucommia-containing preparations. Chin Tradit Pat Med. 2017;39:632-636.

2. Yang F, Dong $X, M a F$, et al. The interventional effects of Tubson-2 Decoction on ovariectomized rats as determined by a combination of network pharmacology and metabolomics. Front Pharmacol. 2020;14(11):581991.

3. Zhao J, Dong C, Shi J, et al. Micro-histomorphometry study of Mongolian medicine echinops sphaerocephalus L. on postmenopausal osteoporosis. Chin J Osteoporosis. 2020;26:972-7.

4. Wang J, Dong X, Ma F, et al. Metabolomics profiling reveals Echinops latifolius Tausch improves the trabecular micro-architecture of ovariectomized rats mainly via intervening amino acids and glycerophospholipids metabolism. J Ethnopharmacol. 2020;260:113018.

5. Wang J. Study on the mechanism of Mongolian medicine Nitraria glabra against osteoporosis based on network pharmacology [D]. Inner Mongolia Medical University, 2019.

6. Gong X, Luan Q, Zhou X, Zhao Y, Zhao C. UHPLC-ESI-MS/MS determination and pharmacokinetics of pinoresinol glucoside and chlorogenic acid in rat plasma after oral administration of Eucommia ulmoides Oliv extract. Biomed Chromatogr. 2017;11:31(11).

7. Wang C, Li T, He J, Li J, Wang Y. Ethnobotany, phytochemistry and pharmacological properties of eucommia ulmoides: a review. Am J Chin Med. 2019;47(2):259-300.

8. He M, Jia J, Li J, et al. Application of characteristic ion filtering with ultra-high performance liquid chromatography quadrupole time of flight tandem mass spectrometry for rapid detection and identification of chemical profiling in Eucommia ulmoides. Oliv J Chromatogr A. 2018;15(1554):81-91.

9. Zhang L, Ke T, Tang Z, Chen X, Lu J. Phytochemistry and pharmacology of carthamus tinctorius L. Am J Chin Med. 2016;44(2):197-226.

10. Duan X, Pan L, Peng D, Bao Q, Xiao L, Zhou A. The analysis of the active components and metabolites of taohong siwu decoction based on ultra performance liquid chromatography quadrupole time-of-flight mass spectrometry. J Sep Sci. 2020;43(22):4131-47.

11. Zong X, Zhang H, Li B, Li L. Study on the electrospray multistage tandem mass spectrometry of the pigment compounds in safflower. J Chin Mass Spe. 2012;33(006):357-62.

12. Xie W, Meng X, Zhai Y, et al. Panax Notoginseng Saponins: A Review of Its Mechanisms of Antidepressant or Anxiolytic Effects and Network Analysis on Phytochemistry and Pharmacology. Molecules. 2018;23(4):940.

13. Ju Z, Li J, Lu Q, Yang Y, Yang L, Wang Z. Identification and quantitative investigation of the effects of intestinal microflora on the metabolism and pharmacokinetics of notoginsenoside Fc assayed by liquid chromatography / electrospray ionization tandem mass spectrometry. J Sep Sci. 2019;42(9):1740-9.

14. Ma Y, Huang BX, Tang WW, et al. Characterization of chemical constituents and metabolites in rat plasma after oral administration of San Miao Wan by ultra-high performance liquid chromatography tandem Q-Exactive Orbitrap mass spectrometry. J Chromatogr B. 2021;28(1178):122793.

15. Zhang J, Huang ZH, Qiu XH, Yang YM, Zhu DY, Xu W. Neutral fragment filtering for rapid identification of new diester-diterpenoid alkaloids in roots of Aconitum carmichaeli by ultra-high-pressure liquid chromatography coupled with linear ion trap-orbitrap mass spectrometry. PloS One.

2012;7(12):e52352.

16. Qiao X, Lin XH, Ji S, Zhang Z. X, Bo T, Guo DA. Global profiling and novel structure discovery using multiple neutral loss / precursor ion scanning combined with substructure recognition and statistical analysis (MNPSS): characterization of terpene-conjugated curcuminoids in curcuma longa as a case study. Anal Chem. 2016;88(1):703-10.

17. Waldner BJ, Machalett R, Schönbichler S, et al. Fast evaluation of herbal substance class composition by relative mass defect plots. Anal Chem. 2020;92(19):12909-16.

18. Fu LL, Ding H, Han LF, et al. Simultaneously targeted and untargeted multicomponent characterization of erzhi pill by offline two-dimensional liquid chromatography / quadrupole - orbitrap mass spectrometry. J Chromatogr A. 2019;11(1584):87-96.

19. He LL, Jiang H, Lan TH, Qiu Y, et al. Chemical profile and potential mechanisms of huo-tan-chu-shi decoction in the treatment of coronary heart disease by UHPLC-Q/TOF-MS in combination with network pharmacology analysis and experimental verification. J Chromatogr B. 2021;15(1175):122729.

20. Liu XG, Li JS, Feng S. X, et al. A high-resolution MS /MS based strategy to improve xenobiotic metabolites analysis by metabolic pathway extension searching combined with parallel reaction monitoring: Flavonoid metabolism in wound site as a case. J Chromatogr B. 2021;1(1162):122470.

Page 20/25 
21. Li Y, Zhang Q. Structural characterisation and screening of triterpene saponins in the bark of llex rotunda using high-performance liquid chromatography coupled to electrospray ionisation and quadrupole time-of-flight mass spectrometry. Phytochem Anal. 2021;32(3):395-403.

22. Yoshikawa M, Morikawa T, Yashiro K, Murakami T, Matsuda H. Bioactive saponins and glycosides. XIX. Notoginseng(3): immunological adjuvant activity of notoginsenosides and related saponins: structures of notoginsenosides- $L,-M$, and $-\mathrm{N}$ from the roots of Panax notoginseng (Burk.) F. $H$. Chen. Chem Pharm. 2001;49(11):1452-6.

23. Yang WZ, Shi XJ, Yao CL, et al. A novel neutral loss / product ion scan-incorporated integral approach for the untargeted characterization and comparison of the carboxyl-free ginsenosides from panax ginseng, panax quinquefolius, and panax notoginseng. J Pharm Biomed Anal. 2020;5(177):112813.

24. Vanholme B, El Houari I, Boerjan W. Bioactivity: phenylpropanoids' best kept secret. Curr Opin Biotechnol. 2019;56:156-162.

25. Wang X, Wu WY, Zhang J. Q, et al. An integrated strategy for holistic quality identification of Chinese patent medicine: Liuwei Dihuang Pills as a case study. Phytochem Anal. 2021;32(2):183-97.

26. Wang X, Wu CT, Xu M, et al. Optimisation for simultaneous determination of iridoid glycosides and oligosaccharides in Radix Rehmannia by microwave assisted extraction and HILIC-UHPLC-TQ-MS/MS. Phytochem Anal. 2020;31(3):340-8.

27. He MZ, Jia J, Li JM, et al. Application of characteristic ion filtering with ultra-high performance liquid chromatography quadrupole time of flight tandem mass spectrometry for rapid detection and identification of chemical profiling in Eucommia ulmoides Oliv. J Chromatogr A. 2018;15(1554):81-91.

28. Shi FY, Tong CY, He CX, et al (2021) Diagnostic ion filtering targeted screening and isolation of anti-inflammatory iridoid glycosides from Hedyotis diffusa. J Sep Sci 22.

29. Sani TH, Hadjmohammadi M, Fatemi MH. Extraction and determination of flavonoids in fruit juices and vegetables using $\mathrm{Fe}_{3} \mathrm{O}_{4} / \mathrm{SiO}_{2} \mathrm{magnetic}$ nanoparticles modified with mixed hemi / admicelle cetyltrimethylammonium bromide and high performance liquid chromatography. J Sep Sci. 2020;43(7):1224-31.

30. Yang PJ, Zhou MD, Zhou C. Y, et al. Separation and purification of both tea seed polysaccharide and saponin from camellia cake extract using macroporous resin. J Sep Sci. 2015;38:656-62.

31. Teich M, Schmidtpott M, Pinxteren DV, Chen JM, Herrmann H. Separation and quantification of imidazoles in atmospheric particles using LC-Orbitrap-MS. J Sep Sci. 2020;43:577-89.

32. Li N, Dong X, Ma FX. Pharmacokinetics study of 16 active ingredients from Tabson-2 decoction in normal and d-galactose induced osteoporosis rats by liquid chromatography-tandem mass spectrometry. J Sep Sci. 2020;43:3702-13.

\section{Figures}



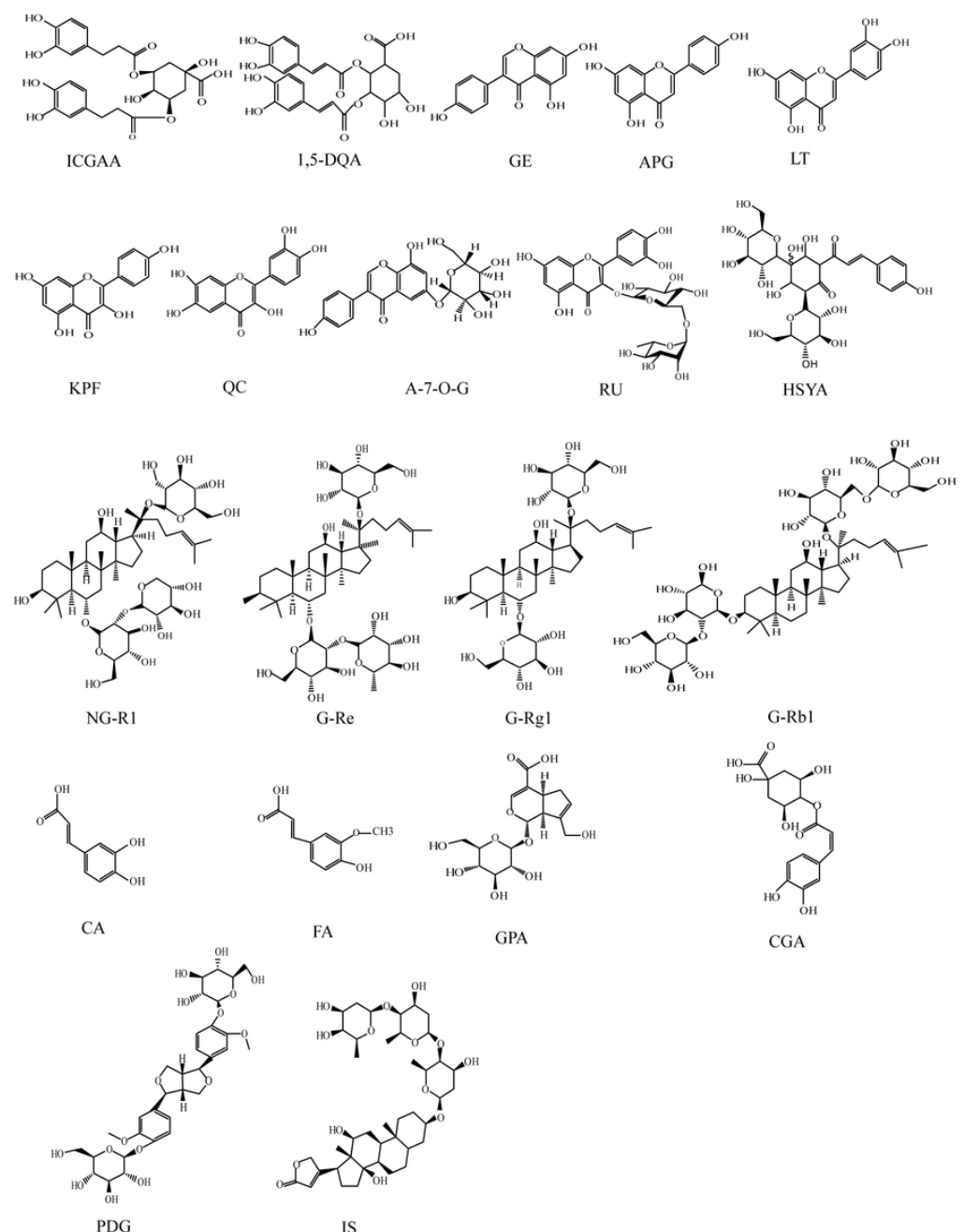

\section{Figure 1}

The chemical structures of nineteen analystes. 


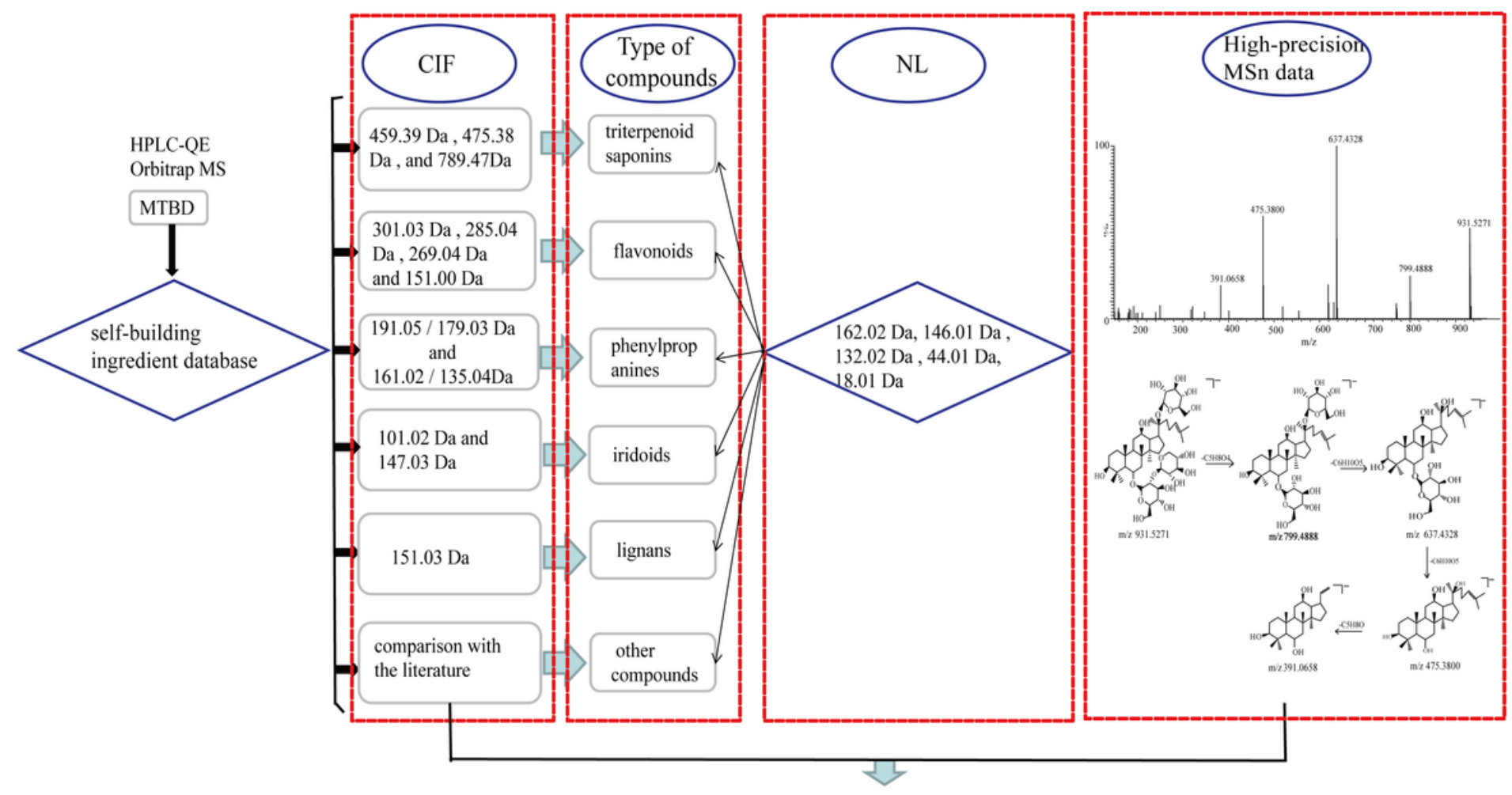

The most possible structure

Figure 2

Analysis strategy of qualitative research of MTBD. 
A

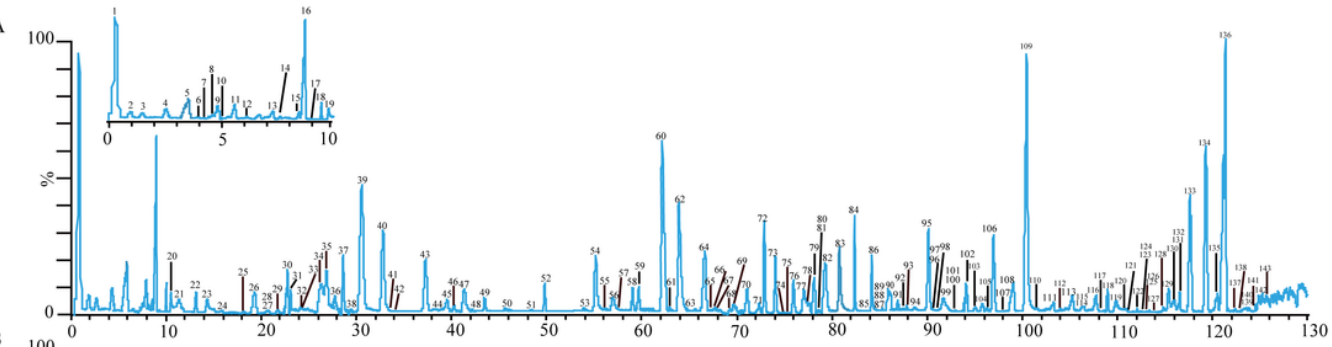

B

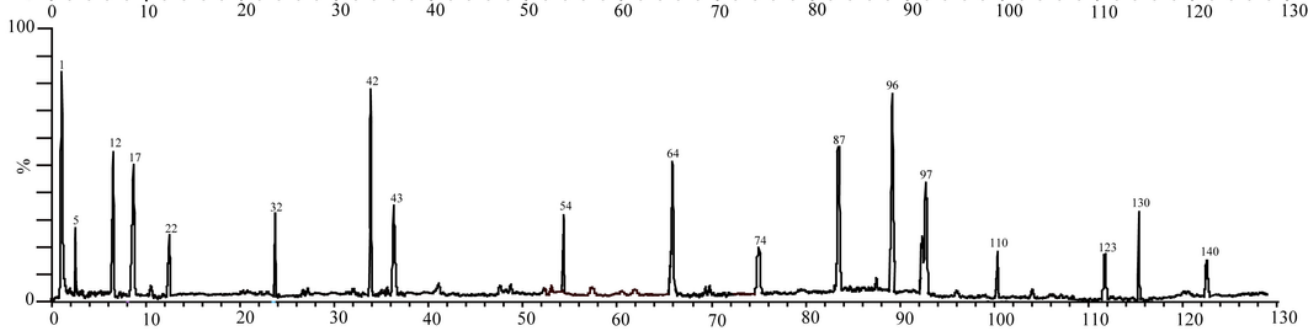

$\mathrm{C}$
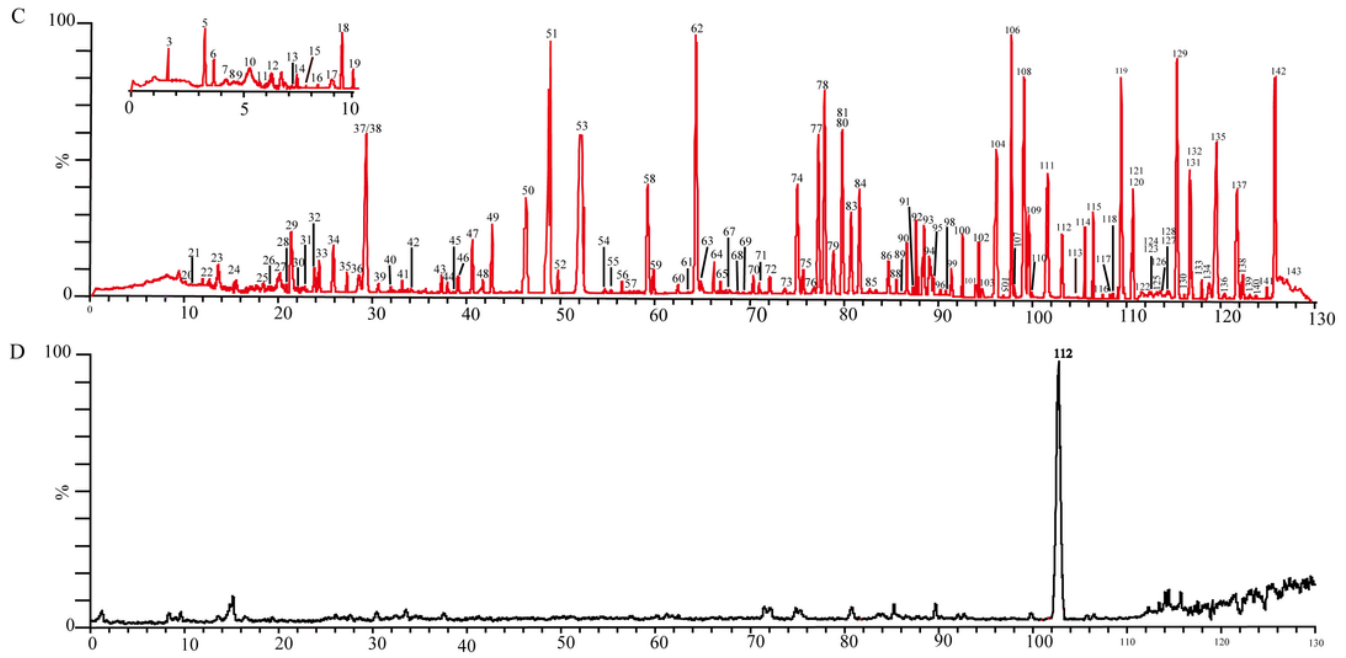

Figure 3

The typical total ion chromatograms (TICs) of MTBD. A: TIC in negative ion mode. B: Comparison with standard in negative ion mode. C: TIC in positive ion mode. D: Comparison with standard in positive ion mode. 
A
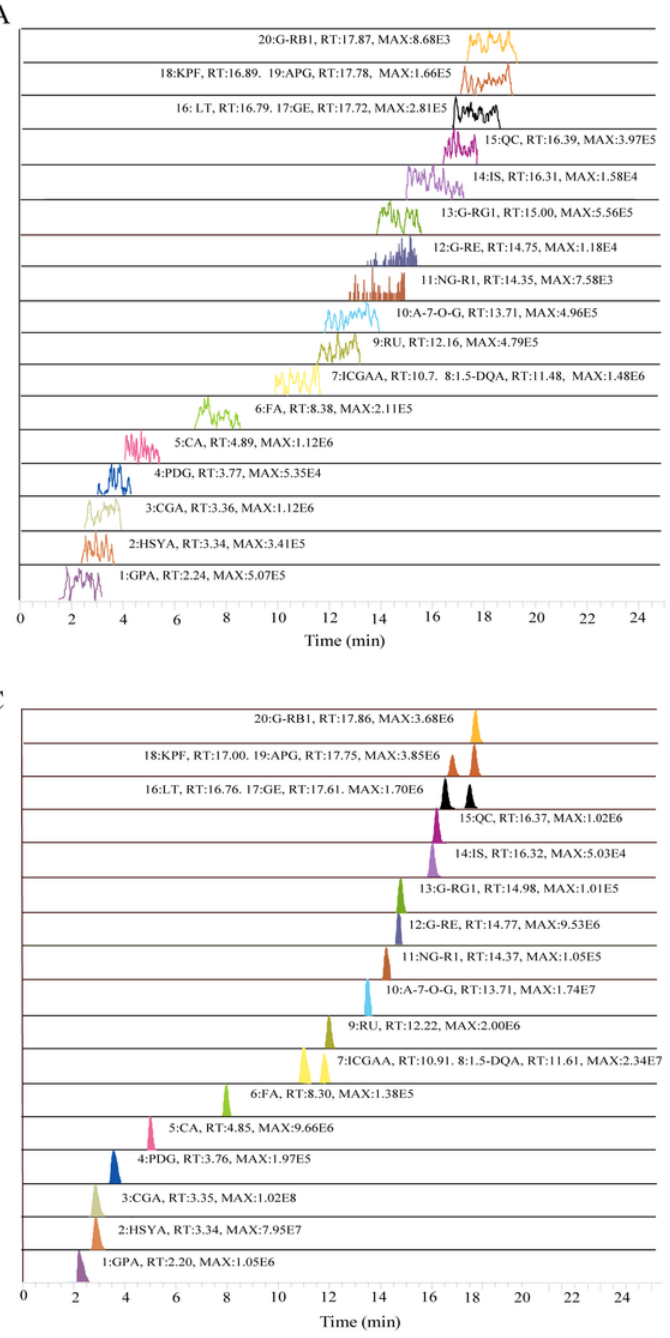

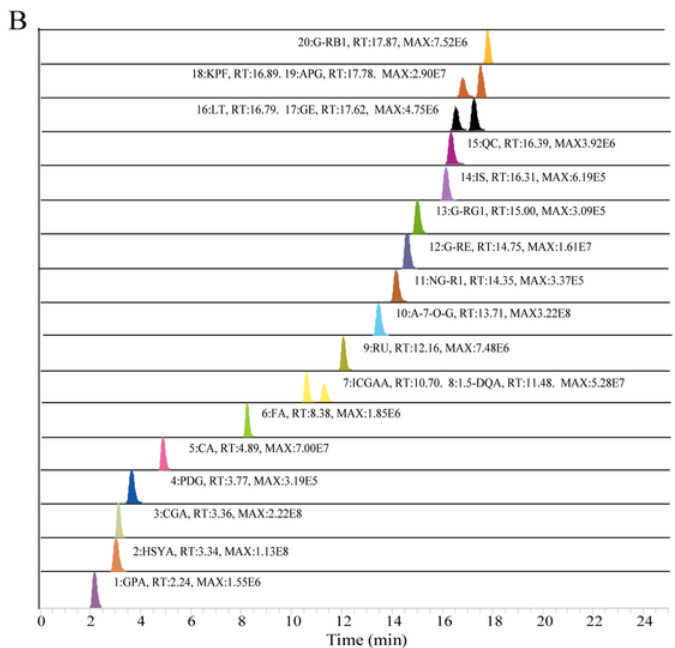

\section{Figure 4}

Representative chromatograms of (A) blank, (B) 19 standard sample, and (C) 19 compounds in MTBD

\section{Supplementary Files}

This is a list of supplementary files associated with this preprint. Click to download.

- Supplementarymaterial.docx 